\title{
Consistent approximate models of the global atmosphere: shallow, deep, hydrostatic, quasi-hydrostatic and non-hydrostatic*
}

\author{
A. A. White ${ }^{1 \dagger}$, B. J. Hoskins ${ }^{2}$, I. Roulstone ${ }^{1,3}$ and A. Staniforth ${ }^{1}$ \\ ${ }^{1}$ Met Office, Exeter, UK \\ ${ }^{2}$ Department of Meteorology, University of Reading, UK \\ ${ }^{3}$ Department of Mathematics and Statistics, University of Surrey, UK \\ Revised: 5th January, 2005
}

${ }^{*}$ (C) Crown copyright, 2004

${ }^{\dagger}$ Corresponding author address: Met Office, FitzRoy Road, Exeter, Devon EX1 3PB, UK 


\section{SUMMARY}

We study global atmosphere models that are at least as accurate as the hydrostatic primitive equations (HPEs), reviewing known results and reporting some new ones. The HPEs make spherical geopotential and shallow atmosphere approximations in addition to the hydrostatic approximation. As is well known, a consistent application of the shallow atmosphere approximation requires omission of those Coriolis terms that vary as the cosine of latitude and of certain other terms in the components of the momentum equation. An approximate model is here regarded as consistent if it formally preserves conservation principles for axial angular momentum, energy and potential vorticity, and (following R. Müller) if its momentum component equations have Lagrange's form. Within these criteria, four consistent approximate global models, including the HPEs themselves, are identified in a height-coordinate framework. The four models, each of which includes the spherical geopotential approximation, correspond to whether the shallow atmosphere and hydrostatic (or quasi-hydrostatic) approximations are individually made or not made. Restrictions on representing the spatial variation of apparent gravity occur. Solution methods and the situation in a pressure-coordinate framework are discussed.

KEYWORDS:

Apparent gravity

Conservation properties

Coriolis force

Lagrange's equations

Primitive equations 


\section{Introduction}

Most global weather forecasting and climate models are based on the hydrostatic primitive equations (HPEs). The HPEs describe both gravity-wave and nearly-geostrophic motion, and much effort has been put into deriving approximate forms that treat only the nearlygeostrophic or "balanced" motion; see Norbury \& Roulstone (2002) for reviews. However, the HPEs themselves include at least three simplifications: as well as the hydrostatic assumption, they embody certain spherical geopotential and shallow atmosphere approximations.

The spherical geopotential approximation is essentially geometric; a subsidiary approximation is required (as we shall argue) but it is quantitatively minor. The shallow atmosphere approximation is more subtle. It involves subsidiary approximations not overtly associated with shallowness - primarily the omission from the components of the momentum equation of those Coriolis terms that vary as the cosine of the latitude $(\cos \phi)$. Following Eckart (1960), this omission has been called the "traditional approximation".

The $\cos \phi$ Coriolis terms have been the subject of quiet controversy in meteorology and oceanography for many years. Linearised, adiabatic analyses [Phillips (1968), Phillips (1990), Thuburn et al. (2002a)] suggest that the terms are not important given typical terrestrial values $(\ll 1)$ of the ratio $\Re$ of planetary rotation frequency to buoyancy frequency, and this is borne out by the similarity of numerical integrations with and without the terms [R. A. Bromley, private communication, T. Davies, private communication]. But since buoyancy frequencies vary widely, and the global circulation is driven by diabatic processes, the neglect of the terms is unsettling. This is especially so if conditions differ markedly from those currently prevailing on the Earth (either in a climate change scenario or because a different planetary atmosphere is being studied). Further, thought experiments can be devised in which omission of the terms leads to small but not negligible effects (White \& Bromley (1995)). The importance of the terms near the equator is suggested by the analyses of Bretherton (1964) and Colin de Verdière \& Schopp (1994), and for mesoscale motion by Draghici (1989). Their retention has been associated with the occurrence of novel nearinertial modes in linearised analyses framed in Cartesian geometry (Thuburn et al. (2002b), Kasahara (2003a), Kasahara (2003b); see also Egger (1999)). Because of these indications, a quasi-hydrostatic formulation that includes the $\cos \phi$ Coriolis terms in a consistent fashion was used as the basis of the Met Office's Unified Model from 1992 until its replacement by an even more comprehensive non-hydrostatic formulation in 2002 (Cullen (1993), White \& Bromley (1995), Staniforth (2001), Davies et al. (2004)).

The development of non-hydrostatic models for global simulation has had three main motivations. First, as spatial resolutions become finer, the use of the hydrostatic approximation becomes inappropriate at the smallest resolved scales (Daley (1988)), yet the use of a single equation set to describe all scales remains desirable. Second, given the availability of semi-implicit methods to handle acoustic waves efficiently, the use of the more accurate nonhydrostatic equations has become a practical proposition (Tanguay et al. (1990)). Third, the mathematical pedigree of the HPEs is less well established than that of the non-hydrostatic equations (which are essentially the usual equations of classical compressible fluid dynamics). In practice, the use of non-hydrostatic dynamics at global NWP centres has proceeded both with and without the inclusion of deep atmosphere effects: for example, the Meteorological Service of Canada has retained the shallow atmosphere approximation (Yeh et al. (2002)) 
while the UK Met Office has included deep atmosphere effects (Staniforth (2001)).

In the deep oceans the buoyancy frequency is typically an order of magnitude less than in the atmosphere (see Gill (1982), p52), so the $\cos \phi$ Coriolis terms are correspondingly more important because the ratio $\Re$ is larger. The ocean model of Marshall et al. (1997) can include in a consistent fashion all of the Coriolis terms as well as non-hydrostatic effects.

In this paper we examine how deep/shallow and hydrostatic/non-hydrostatic formulation elements may be combined in a consistent way. We regard a model as being consistent if it implies axial angular momentum, energy and potential vorticity conservation principles analogous to those of the unapproximated equations, and - following Müller (1989) - if its component momentum equations have the form of Lagrange's equations.

Approximations are inevitably made when continuous equations are discretized in the construction of a numerical model. The governing equations are known to deliver (to an extent depending on the initial state) time evolutions that are sensitive to changes in the initial state - to behave "chaotically". The representation of forcing processes such as diabatic heating and cooling is subject to considerable uncertainty, especially in climate simulation. Our objective in formulating consistent continuous approximate models is to enable these challenging issues to be confronted without the suspicion that discretized model behaviour might partly reflect some unphysical property of the underlying continuous formulation.

All of the approximate models that we discuss involve the spherical geopotential approximation. This approximation is discussed in Section 2. The four consistent models noted by Staniforth (2001) are surveyed in a height-coordinate framework in section 3; for overviews, see Figure 4 and Table 1. Section 4 considers the time integration of these models and discusses the situation in pressure coordinates. Conclusions are given in section 5. The Appendices present mathematical material in support of the main sections.

\section{The spherical geopotential approximation}

In terms of the field of velocity $\mathbf{u}$ measured in a frame $\Im$ rotating with uniform angular velocity $\Omega$ relative to an inertial frame, the momentum equation can be written as

$$
\frac{D \mathbf{u}}{D t} \equiv\left(\frac{\partial}{\partial t}+\mathbf{u} \cdot \nabla\right) \mathbf{u}=-2 \boldsymbol{\Omega} \times \mathbf{u}-\boldsymbol{\Omega} \times(\boldsymbol{\Omega} \times \mathbf{r})-\nabla \Phi_{N}-\alpha \nabla p+\mathbf{G} .
$$

Here $D / D t$ is the material derivative in the frame $\Im, \mathbf{r}$ is position relative to any fixed origin on the axis of rotation of $\Im, \nabla$ is the 3-dimensional gradient operator, $\Phi_{N}$ is Newtonian gravitational potential, $\alpha$ is specific volume and $p$ is pressure. The terms on the right-hand side represent the forces acting (per unit mass): Coriolis $(-2 \boldsymbol{\Omega} \times \mathbf{u})$, centrifugal $(-\boldsymbol{\Omega} \times(\boldsymbol{\Omega} \times \mathbf{r}))$, Newtonian gravity $\left(-\nabla \Phi_{N}\right)$, pressure gradient $(-\alpha \nabla p)$, and all other forces $(\mathbf{G})$.

For discussion of (2.1) as an expression of Newton's second law of motion see, for example, Lorenz (1967), Phillips (1973), Gill (1982), Pedlosky (1987), Holton (1992) and Dutton (1995). As described in these references, it is usual to combine the centrifugal force $(-\boldsymbol{\Omega} \times$ $(\boldsymbol{\Omega} \times \mathbf{r}))$ with Newtonian gravity $\left(-\nabla \Phi_{N}\right)$ to give apparent gravity, $-\nabla \Phi_{A}$, where $\Phi_{A}=$ $\Phi_{N}-\Omega^{2} s^{2} / 2$ is a new scalar potential, $s$ being perpendicular distance from the axis of rotation of $\Im$. Equation (2.1) then condenses to

$$
\frac{D \mathbf{u}}{D t}=-2 \boldsymbol{\Omega} \times \mathbf{u}-\nabla \Phi_{A}-\alpha \nabla p+\mathbf{G} .
$$


From (2.2) many further relations may be derived. For example, $\mathbf{r} \times(2.2)$ and use of the kinematic relation $\mathbf{u}=D \mathbf{r} / D t$ leads to an angular momentum equation:

$$
\frac{D}{D t}(\mathbf{r} \times \mathbf{u})=\mathbf{r} \times\left(-2 \mathbf{\Omega} \times \mathbf{u}-\nabla \Phi_{A}-\alpha \nabla p+\mathbf{G}\right)
$$

By forming the scalar product of (2.3) with unit vector $\widehat{\Omega}$ in the direction of the vector $\boldsymbol{\Omega}$ (here assumed constant), an axial absolute angular momentum equation can be obtained:

$$
\frac{D}{D t}[\widehat{\boldsymbol{\Omega}} \cdot(\mathbf{r} \times(\mathbf{u}+\mathbf{\Omega} \times \mathbf{r}))]=\widehat{\mathbf{\Omega}} \cdot\left[\mathbf{r} \times\left(-\nabla \Phi_{A}-\alpha \nabla p+\mathbf{G}\right)\right] .
$$

Taking the curl $(\nabla \times)$ of $(2.2)$ and applying standard vector differential identities gives a vorticity equation in the form

$$
\frac{D \mathbf{Z}}{D t}+\mathbf{Z} \nabla \cdot \mathbf{u}-(\mathbf{Z} \cdot \nabla) \mathbf{u}=\nabla p \times \nabla \alpha+\nabla \times \mathbf{G}
$$

in which $\mathbf{Z}=2 \boldsymbol{\Omega}+\nabla \times \mathbf{u}$ is the absolute vorticity, and $\nabla \cdot$ denotes the divergence of the vector operand. See Pedlosky (1987) and Dutton (1995).

Other relations follow upon use of the continuity and thermodynamic equations:

$$
\begin{aligned}
& \frac{D \rho}{D t}+\rho \nabla \cdot \mathbf{u}=0 \\
& \frac{D \theta}{D t}=\left(\frac{\theta}{T c_{p}}\right) Q
\end{aligned}
$$

Here $\rho=1 / \alpha$ is density, $T$ is temperature, $c_{p}$ is specific heat at constant pressure, $Q$ is the diabatic heating rate per unit mass and $\theta$ is the potential temperature (given by $T\left(p_{\text {ref }} / p\right)^{R / c_{p}}, R$ being the gas constant per unit mass of air and $p_{\text {ref }}$ a constant pressure conventionally taken as $1000 \mathrm{hPa}$ ). Perfect gas behaviour, i.e. $p=\rho R T$, is assumed. From (2.2), (2.6) and (2.7) an energy conservation law readily results:

$$
\rho \frac{D}{D t}\left(\frac{1}{2} \mathbf{u}^{2}+\Phi_{A}+c_{v} T\right)+\nabla \cdot(p \mathbf{u})=\rho(Q+\mathbf{u} \cdot \mathbf{G})
$$

( $c_{v}=c_{p}-R$ is specific heat at constant volume.) From (2.5), (2.6) and (2.7) a conservation law for Ertel's potential vorticity $\alpha \mathbf{Z} \cdot \nabla \theta$ follows:

$$
\rho \frac{D}{D t}(\alpha \mathbf{Z} \cdot \nabla \theta)=\nabla \cdot\left(\mathbf{Z} \frac{D \theta}{D t}+\theta \nabla \times \mathbf{G}\right) .
$$

Potential vorticity remains unchanged following the flow if the right-hand side of (2.9) vanishes, and in particular if the motion is isentropic and $\mathbf{G}=0 . \mathrm{Eq}$ (2.9) is Ertel's theorem; see Gill (1982), Pedlosky (1987) and Dutton (1995).

The governing equations and the relations that follow from them are vector invariant forms that hold irrespective of coordinate system. But the construction of a numerical model based on the governing equations requires the choice of a coordinate system and the 
separation of vector equations into components. The issue of approximation then arises because of the geometry of the surfaces of constant apparent gravity potential, $\Phi_{A}$.

By definition, the direction of apparent gravity $\left(-\nabla \Phi_{A}\right)$ is normal to surfaces of constant $\Phi_{A}$. These surfaces - called geopotential surfaces, or simply geopotentials - are surfaces of constant $\left(\Phi_{N}-\Omega^{2} s^{2} / 2\right)$, and they are not spheres; see Figure 1. Separating (2.2) into components within and perpendicular to geopotentials is mathematically and physically desirable because $\Phi_{A}$ then appears only in the perpendicular component, whose direction coincides with the apparent vertical seen by an observer in the rotating frame. If done exactly, the separation takes account of the departure of the geopotentials from sphericity. However, as described in the references already cited, the departure from sphericity is sufficiently small given terrestrial parameter values that a spherical geopotential approximation is usually invoked $^{1}$ : the components of (2.2) within and perpendicular to geopotentials are sought, but the geopotentials, as geometric entities, are treated as spheres once the separation has been made. This procedure is not the same as isolating the components of (2.2) in a spherical polar coordinate system; in that case, terms involving $\Phi_{A}$ would occur in the meridional component as well as the radial component, and they would not be negligible. Neither would the Earth's surface be a coordinate surface, even in the absence of mountains.

To develop an analytical framework, we examine the components of (2.2) in Lagrange's form in a general orthogonal coordinate system. This approach is inspired by the concise treatment of Müller $(1989)^{2}$, but differs from it by working throughout in terms of velocities relative to the rotating frame, and is motivated partly by the formal similarity between the Coriolis force and the Lorentz force which acts on a charged particle moving in a magnetic field; see, for example, Sivadière (1983). Details are given in Appendix A. The $i$ th component of (2.2) in the orthogonal coordinate system $q_{i}=q_{i}\left(x_{j}\right)$ [where $x_{j}$ are Cartesian coordinates in the rotating frame and indices $i$ and $j$ both range from 1 to 3] may be written in Lagrange's form as

$$
\frac{D}{D t}\left(\frac{\partial L}{\partial \dot{q}_{i}}\right)-\frac{\partial L}{\partial q_{i}}=-\alpha \frac{\partial p}{\partial q_{i}}+h_{i} G_{i} .
$$

Here $G_{i}$ is the component of $\mathbf{G}$ in the direction of $\nabla q_{i}, h_{i}$ is the corresponding metric factor (see (A.12)) and $\dot{q}_{i} \equiv D q_{i} / D t$; the Lagrangian function $L$ is defined as

$$
L \equiv T-M-\Phi_{A},
$$

where $T$ is the specific relative kinetic energy, i.e.

$$
T \equiv \frac{1}{2} \mathbf{u}^{2},
$$

and $M$, the velocity-dependent potential term of the Coriolis force, is given by

$$
M \equiv-\mathbf{u} \cdot(\boldsymbol{\Omega} \times \mathbf{r})
$$

$L$ is a function of $q_{i}$ and $\dot{q}_{i}$ (but not explicitly of time $t$ ) and the differentiation with respect to $q_{i}$ in $\partial L / \partial q_{i}(\mathrm{Eq}(2.10))$ is taken at constant $\dot{q}_{i}$.

\footnotetext{
${ }^{1}$ The geopotentials could be represented more accurately by spheroids; see Gill (1982) p91. The momentum component equations in an oblate spheroidal coordinate system are lucidly derived by Gates (2004).

${ }^{2}$ As discussed in Appendix A, the method used by Müller (1989) is given in detail in the recent textbook by Zdunkowski \& Bott (2003).
} 
Given any specification of the shape of the geopotentials, (2.10) could be used to derive the components of the momentum equation in an appropriate coordinate system. Limiting attention to cases in which the geopotentials are independent of longitude, we choose a right-handed coordinate system $\left(q_{1}, q_{2}, q_{3}\right)$ in which: (i) $q_{1}$ is longitude; (ii) $\nabla q_{2}$ also lies in geopotential surfaces and is perpendicular to $\nabla q_{1}$, and (iii) $\nabla q_{3}$ is in the direction of apparent vertical (perpendicular to the geopotentials). In the spherical geopotential approximation, $q_{1}$, $q_{2}$ and $q_{3}$ are specified as ordinary spherical polar coordinates, e.g. $q_{1}=\lambda$ (longitude), $q_{2}=\phi$ (latitude) and $q_{3}=r$ (mean radius of the Earth plus height above a reference geopotential surface coinciding with mean sea level), and - crucially - $\Phi_{A}$ is assumed to be a function of $r$ only: $\Phi_{A}=\Phi_{A}(r)$. The polar axis of the coordinate system coincides with the direction of the frame rotation vector $\Omega$, the origin is at the centre of the Earth - see Figure 2 - and we have

$$
\begin{aligned}
T \equiv \frac{1}{2} \mathbf{u}^{2} & =\frac{1}{2}\left[(\dot{\lambda})^{2} r^{2} \cos ^{2} \phi+(\dot{\phi})^{2} r^{2}+(\dot{r})^{2}\right], \\
M & \equiv-\mathbf{u} \cdot(\boldsymbol{\Omega} \times \mathbf{r})=-\dot{\lambda} \Omega r^{2} \cos ^{2} \phi
\end{aligned}
$$

Here $\dot{\lambda} \equiv D \lambda / D t, \dot{\phi} \equiv D \phi / D t$ and $\dot{r} \equiv D r / D t$; they are related to the components $u, v$ and $w$ of $\mathbf{u}$ in the zonal $(\lambda)$, meridional $(\phi)$ and radial $(r)$ directions by the kinematic equations

$$
u=\dot{\lambda} r \cos \phi, \quad v=\dot{\phi} r, \quad w=\dot{r} .
$$

From (2.10), the $\lambda, \phi$ and $r$ components of (2.2) under the spherical geopotential approximation may be obtained - see Appendix A. They are:

$$
\begin{gathered}
\frac{D u}{D t}=-\frac{u w}{r}+\frac{u v}{r} \tan \phi+2 \Omega v \sin \phi-2 \Omega w \cos \phi-\frac{\alpha}{r \cos \phi} \frac{\partial p}{\partial \lambda}+G_{\lambda} ; \\
\frac{D v}{D t}=-\frac{v w}{r}-\frac{u^{2}}{r} \tan \phi-2 \Omega u \sin \phi-\frac{\alpha}{r} \frac{\partial p}{\partial \phi}+G_{\phi} ; \\
\frac{D w}{D t}=\frac{u^{2}}{r}+\frac{v^{2}}{r}+2 \Omega u \cos \phi-g-\alpha \frac{\partial p}{\partial r}+G_{r} .
\end{gathered}
$$

In (2.18), $g \equiv d \Phi_{A} / d r$ is the magnitude of apparent gravity. The material derivative in (2.17) - (2.19) may be expanded as

$$
\frac{D}{D t}=\frac{\partial}{\partial t}+\dot{\lambda} \frac{\partial}{\partial \lambda}+\dot{\phi} \frac{\partial}{\partial \phi}+\dot{r} \frac{\partial}{\partial r}=\frac{\partial}{\partial t}+\frac{u}{r \cos \phi} \frac{\partial}{\partial \lambda}+\frac{v}{r} \frac{\partial}{\partial \phi}+w \frac{\partial}{\partial r} .
$$

The spherical geopotential approximation places a constraint on permissible variations of $g$. Since the geopotentials are treated as spheres, it would be inconsistent to include latitude variation of $g$ because the line integral of $g \mathbf{k}=\nabla \Phi_{A}$, where $\mathbf{k}$ is unit vector normal to the spheres, would then not vanish around circuits in a meridional plane, and there would be spurious sources both of the zonal component of vorticity and of potential vorticity; see Figure 3. (Radial variation of $g$ is not precluded by this argument; see section 3.) Since, at mean sea level, the value of $g$ at the equator is only about $0.5 \%$ less than at the poles, the approximation involved in neglecting the latitude variation of $g$ is likely to be minor.

In the same way, the spherical geopotential approximation requires neglect of variations of apparent gravity with longitude. These occur in reality because of departures from zonal 
symmetry in the mass distribution of the Earth, the atmosphere and the oceans. Such gravity anomalies are geophysically measurable but are considered to be negligible meteorologically. Another effect that is neglected in meteorological applications is time variation of the Earth's rotation vector. This variation is related to small but measurable length-of-day changes and polar motion; see Barnes et al. (1983). A term in $\dot{\Omega} \times \mathbf{r}$ could be included in (2.1) to account for such changes (see Pedlosky (1987), p.17) but quantitatively it would be minute.

\section{A quartet of consistent global models}

Within the spherical geopotential approximation and our stated criteria for consistent formulation, we find four ways of combining (quasi-)hydrostatic or non-hydrostatic dynamics with shallow or deep atmosphere geometry. This is what one might expect, or at least hope for. A simple way of naming the consistent models would be as "non-hydrostatic, shallow", "hydrostatic, shallow", etc. This terminology runs into difficulty because the "hydrostatic, shallow" model is already well-known as the HPEs; also, the "hydrostatic, deep" equations are not strictly hydrostatic (see section 3(d), below) and have justifiably been called the quasi-hydrostatic equations (QHEs). We keep these names for the models that omit the term $D w / D t$ from the vertical component of the momentum equation, but use "non-hydrostatic, deep" (NHD) and "non-hydrostatic shallow" (NHS) for the models that retain $D w / D t$. Figure 4 and Table 1 display key features of the four consistent models.

\section{(a) The non-hydrostatic, deep (NHD) model}

The relevant equations were presented in the previous section: (2.6), (2.7) and (2.16) (2.19), $D / D t$ being given throughout by (2.20). Rather than via the Lagrange route adopted here (in Appendix A), (2.17) - (2.19) are more usually derived by separating the zonal, meridional and vertical components of the momentum equation (2.2) after appeal to the spherical geopotential approximation; see Gill (1982), Holton (1992) and White (2003). With the understanding that they incorporate the spherical geopotential approximation, we call (2.6), (2.7) and (2.16) - (2.20) the non-hydrostatic, deep (NHD) equations.

The six terms on the right-hand sides of (2.17) - (2.19) that are quadratic in the velocity components are the metric or curvature terms; they reflect changes of orientation of the coordinate axes with spatial location on spherical surfaces. Two types of metric term can be recognised. The four not involving $\tan \phi$ reflect the curvature of great circles and hence the intrinsic uniform curvature of the sphere. The two involving $\tan \phi$ reflect the additional curvature of latitude circles. The four terms involving $\Omega$ are the Coriolis terms; two of them vary as $\sin \phi$ and two as $\cos \phi$. The other terms in (2.17) - (2.19) represent components of the pressure gradient force and of the remaining force $\mathbf{G}$.

Equations (2.17) - (2.19) may be written in more compact forms, one of which involves separating factors of $(2 \Omega+u /(r \cos \phi))$ amongst the metric and Coriolis terms on the righthand sides. Some metric terms remain outside this factorization in (2.18) and (2.19), however. A very compact form of (2.17) is the axial angular momentum form (3.1), below.

When the requirement that $g$ vary only with $r$ is enforced (see section 2), the rotation rate $\Omega$ of the frame $\Im$ appears explicitly only in the Coriolis terms. The conservation properties 
of $(2.17)$ - (2.19) are thus those of the vector form (2.2) in which $g \mathbf{k}$ acts radially.

From (2.17) (using (2.16)) an axial angular momentum conservation law is readily derived:

$$
\rho \frac{D}{D t}[(u+\Omega r \cos \phi) r \cos \phi]=\rho G_{\lambda} r \cos \phi-\frac{\partial p}{\partial \lambda} .
$$

This is the spherical polar version of (2.4); the origin is now at the centre of the Earth, and the gravitational torque term has vanished because of the spherical geopotential approximation.

Given the kinematic relations (2.16), the angular momentum law (3.1) is the most compact form of the zonal momentum equation (2.17), and could have been written down immediately. White (2002) gives a reverse derivation of the component equations (2.17) - (2.19) that starts from (3.1) and invokes basic features of the energetics.

Other conservation properties follow from (2.17) - (2.19) in conjunction with the continuity and thermodynamic equations (2.6) and (2.7). From (2.17) - (2.19) and (2.7) an energy conservation law of the form (2.8) results. From (2.17) - (2.19), (2.6) and (2.7) a potential vorticity conservation law of the form (2.9) follows. In these spherical polar forms of (2.8) and (2.9), the vector gradient operator $\nabla$ is given by

$$
\nabla \equiv\left(\frac{1}{r \cos \phi} \frac{\partial}{\partial \lambda}, \frac{1}{r} \frac{\partial}{\partial \phi}, \frac{\partial}{\partial r}\right)
$$

and the divergence $(\nabla \cdot)$ of a vector field $\mathbf{A}=\left(A_{\lambda}, A_{\phi}, A_{r}\right)$ by

$$
\nabla \cdot \mathbf{A} \equiv \frac{1}{r \cos \phi}\left(\frac{\partial A_{\lambda}}{\partial \lambda}+\frac{\partial}{\partial \phi}\left(A_{\phi} \cos \phi\right)\right)+\frac{1}{r^{2}} \frac{\partial}{\partial r}\left(r^{2} A_{r}\right)
$$

The curl $(\nabla \times)$ of $\mathbf{A}$ is given in component form as

$$
\nabla \times \mathbf{A} \equiv \frac{1}{r}\left(\frac{\partial A_{r}}{\partial \phi}-\frac{\partial}{\partial r}\left(r A_{\phi}\right), \frac{\partial}{\partial r}\left(r A_{\lambda}\right)-\frac{1}{\cos \phi} \frac{\partial A_{r}}{\partial \lambda}, \frac{1}{\cos \phi}\left[\frac{\partial A_{\phi}}{\partial \lambda}-\frac{\partial}{\partial \phi}\left(A_{\lambda} \cos \phi\right)\right]\right)
$$

The components of the absolute vorticity $\mathbf{Z} \equiv \nabla \times \mathbf{u}+2 \boldsymbol{\Omega}$ in the spherical polar $\lambda, \phi, r$ system are thus

$$
\begin{gathered}
Z_{\lambda} \equiv \frac{1}{r} \frac{\partial w}{\partial \phi}-\frac{1}{r} \frac{\partial}{\partial r}(r v), \\
Z_{\phi} \equiv \frac{1}{r} \frac{\partial}{\partial r}(r u)-\frac{1}{r \cos \phi} \frac{\partial w}{\partial \lambda}+2 \Omega \cos \phi \\
Z_{r} \equiv \frac{1}{r \cos \phi}\left[\frac{\partial v}{\partial \lambda}-\frac{\partial}{\partial \phi}(u \cos \phi)\right]+2 \Omega \sin \phi .
\end{gathered}
$$

The expressions (3.2) - (3.7) are of course well known in a general physics context. As we shall find, they are all modified when the shallow atmosphere approximation is made.

With various extensions to describe and accommodate the presence of water substance in the atmosphere, the NHD equations are the basis of the dynamical core of the Met Office's current Unified Model for weather forecasting and climate simulation (Davies et al. (2004)). They are also the basis of the NICAM formulation described by Tomita \& Satoh (2004). 
Since radial variation of $g$ does not give rise to spurious vorticity sources (see section 2), the question arises as to what radial variation should be adopted in the NHD model. In the Met Office's implementation a constant value has been used. Although the quantitative difference in performance would probably be very small, it can be argued that $g$ should vary as $1 / r^{2}$ to ensure that the implied equation for the tendency $(\partial / \partial t)$ of the 3-dimensional divergence $\nabla \cdot \mathbf{u}$ does not contain a spurious term originating from $\nabla \cdot \nabla \Phi_{A}$ (as defined by (3.3)). This would also remove the bizarre implication that the mass of the Earth depends on the radius of the (larger) sphere over which the flux of the gravitational field is integrated!

\section{(b) The hydrostatic primitive equations (HPEs)}

In a purely formal sense, the momentum components of the hydrostatic primitive equations (HPEs) may be obtained from their NHD counterparts (2.17) - (2.19) by making various changes and omissions in addition to neglect of the term $D w / D t$ :

(i) replacing $r$ by $a$, the mean radius of the Earth, and $\partial / \partial r$ by $\partial / \partial z$, where $z$ is height above mean sea level;

(ii) omitting all the metric terms not involving $\tan \phi$;

(iii) omitting those Coriolis terms that vary as the cosine of the latitude;

(iv) neglecting vertical (as well as horizontal) variation of $g$;

(v) neglecting the vertical component of $\mathbf{G}$, i.e. setting $\mathbf{G}=\mathbf{G}_{h} \equiv\left(G_{\lambda}, G_{\phi}, 0\right)$.

Of these, (i) is the basic shallow atmosphere approximation (including a simple change of vertical coordinate), while (ii) and (iii) are subsidiary approximations required in order to produce the conservation properties and Lagrange's form noted below. (iii) amounts to neglecting the horizontal component of the Earth's rotation $\Omega$ (which at the equator is the sole component of $\boldsymbol{\Omega}$ ). Whether the name shallow atmosphere approximation is applied to (i) or to (i) - (iii) is a matter of taste; we prefer the second option.

The neglect of $D w / D t$ is justified if the time-scale of the motion is much larger than the reciprocal of the buoyancy frequency (see White (2002)). The replacement (i) is justified by the shallowness of the atmosphere in relation to $a$, as is omission (iv) (which also avoids the generation of spurious divergence tendencies when the shallow atmosphere definition (3.17), below, is adopted). The omission (ii) may be justified by considering typical orders of magnitude (see, for example, Holton (1992), section 2.4). The omission (iii) of the $\cos \phi$ Coriolis terms was considered in section 1; see the references cited there, also the discussions in Marshall et al. (1997) and White (2003). (v) is an independent simplification motivated by a desire to reduce (2.19) to the familiar hydrostatic relation, and by the insignificance of friction in the vertical momentum balance on all except the smallest horizontal scales.

The HPEs corresponding to (2.17) - (2.20) are

$$
\begin{gathered}
\frac{D_{a} u}{D t}=\frac{u v}{a} \tan \phi+2 \Omega v \sin \phi-\frac{\alpha}{a \cos \phi} \frac{\partial p}{\partial \lambda}+G_{\lambda} \\
\frac{D_{a} v}{D t}=-\frac{u^{2}}{a} \tan \phi-2 \Omega u \sin \phi-\frac{\alpha}{a} \frac{\partial p}{\partial \phi}+G_{\phi} \\
g+\alpha \frac{\partial p}{\partial z}=0
\end{gathered}
$$


in which the material derivative is now the shallow atmosphere form

$$
\frac{D_{a}}{D t}=\frac{\partial}{\partial t}+\frac{u}{a \cos \phi} \frac{\partial}{\partial \lambda}+\frac{v}{a} \frac{\partial}{\partial \phi}+w \frac{\partial}{\partial z}=\frac{\partial}{\partial t}+\mathbf{u} \cdot \nabla_{a}
$$

The kinematic relations (2.16) also appear in shallow atmosphere forms:

$$
u=\dot{\lambda} a \cos \phi, \quad v=\dot{\phi} a, \quad w=\dot{z} .
$$

As outlined in Appendix A, (3.8) - (3.10) may be derived from Lagrange's equations (2.10) by setting $D / D t \rightarrow D_{a} / D t$ and re-defining $T$ and $M$ (see (2.11), (2.14) and (2.15)) as

$$
\begin{gathered}
T_{H P E} \equiv \frac{1}{2}\left[(\dot{\lambda})^{2} a^{2} \cos ^{2} \phi+(\dot{\phi})^{2} a^{2}\right]=\frac{1}{2} \mathbf{v}^{2}, \\
M_{H P E} \equiv-\dot{\lambda} \Omega a^{2} \cos ^{2} \phi .
\end{gathered}
$$

Here $\mathbf{v}$ is the horizontal flow $(u, v, 0)$. These changes correspond to (i) omitting the contribution of $w$ to the kinetic energy, and (ii) making the shallow atmosphere approximation in the overt form $r \rightarrow a$ in both the kinetic energy and the Coriolis potential.

The thermodynamic equation of the HPEs is the shallow atmosphere version of (2.7):

$$
\frac{D_{a} \theta}{D t}=\left(\frac{\theta}{T c_{p}}\right) Q
$$

In the continuity equation, the definition of divergence is modified as well; $(2.6)$ is replaced by

$$
\frac{D_{a} \rho}{D t}+\rho \nabla_{a} \cdot \mathbf{u}=0
$$

where $\nabla_{a} \cdot \mathbf{u}$ follows the shallow atmosphere rule that, for vector field $\mathbf{A}=\left(A_{\lambda}, A_{\phi}, A_{z}\right)$,

$$
\nabla_{a} \cdot \mathbf{A} \equiv \frac{1}{a \cos \phi}\left(\frac{\partial A_{\lambda}}{\partial \lambda}+\frac{\partial}{\partial \phi}\left(A_{\phi} \cos \phi\right)\right)+\frac{\partial A_{z}}{\partial z}
$$

Given the modifications (3.11), (3.12) and (3.17) of material derivative, kinematic relations and 3D divergence, the HPEs (3.8) - (3.10), (3.15) and (3.16) imply analogues of the NHD axial angular momentum and energy conservation laws (3.1) and (2.8) in the forms

$$
\begin{gathered}
\rho \frac{D_{a}}{D t}[(u+\Omega a \cos \phi) a \cos \phi]=\rho G_{\lambda} a \cos \phi-\frac{\partial p}{\partial \lambda} \\
\rho \frac{D_{a}}{D t}\left(\frac{1}{2} \mathbf{v}^{2}+g z+c_{v} T\right)+\nabla_{a} \cdot(p \mathbf{u})=\rho\left(Q+\mathbf{v} \cdot \mathbf{G}_{h}\right) .
\end{gathered}
$$

The variation of planetary angular momentum with height is not represented in (3.18) (cf. (3.1)). Newton (1971), following Lorenz (1967) (see p 53), found that this height variation is "not quite negligible" in evaluations of the atmosphere's angular momentum budget in low latitudes. White \& Bromley (1995) noted that neglect of the height variation of planetary angular momentum leads to zonal wind errors of up to $2 \mathrm{~ms}^{-1}$ when a parcel of air moves radially from ground level to tropopause in the absence of zonal forces. Eq. (3.19) - in which 
$\mathbf{G}_{h} \equiv\left(G_{\lambda}, G_{\phi}, 0\right)$ - shows that only the horizontal flow $\mathbf{v}$ contributes to the kinetic energy in the HPEs. This well known result is consistent with (3.13) .

The HPEs imply the potential vorticity conservation law (2.9) in the modified form

$$
\rho \frac{D_{a}}{D t}\left(\alpha \mathbf{Z}_{H P E} \cdot \nabla_{a} \theta\right)=\nabla_{a} \cdot\left(\mathbf{Z}_{H P E} \frac{D_{a} \theta}{D t}+\theta \nabla_{a} \times \mathbf{G}_{h}\right) .
$$

The components of the shallow atmosphere gradient operator $\nabla_{a}$ are given by

$$
\nabla_{a} \equiv\left(\frac{1}{a \cos \phi} \frac{\partial}{\partial \lambda}, \frac{1}{a} \frac{\partial}{\partial \phi}, \frac{\partial}{\partial z}\right) \quad\left(\equiv \nabla_{a h}+\left(0,0, \frac{\partial}{\partial z}\right)\right)
$$

and for any vector field $\mathbf{A} \equiv\left(A_{\lambda}, A_{\phi}, A_{z}\right)$ the shallow atmosphere curl, $\nabla_{a} \times \mathbf{A}$, is

$$
\nabla_{a} \times \mathbf{A} \equiv\left(\frac{1}{a} \frac{\partial A_{z}}{\partial \phi}-\frac{\partial A_{\phi}}{\partial z}, \frac{\partial A_{\lambda}}{\partial z}-\frac{1}{a \cos \phi} \frac{\partial A_{z}}{\partial \lambda}, \frac{1}{a \cos \phi}\left[\frac{\partial A_{\phi}}{\partial \lambda}-\frac{\partial}{\partial \phi}\left(A_{\lambda} \cos \phi\right)\right]\right) .
$$

The HPE absolute vorticity $\mathbf{Z}_{H P E}$ is

$$
\mathbf{Z}_{H P E} \equiv \nabla_{a} \times \mathbf{v}+2 \Omega \mathbf{k} \sin \phi=\nabla_{a} \times(\mathbf{v}+\mathbf{i} \Omega a \cos \phi)
$$

in which (from $(3.22)$ and $\mathbf{v} \equiv(u, v, 0)$ ) the HPE relative vorticity $\nabla_{a} \times \mathbf{v}$ is given by

$$
\nabla_{a} \times \mathbf{v} \equiv\left(-\frac{\partial v}{\partial z}, \frac{\partial u}{\partial z}, \frac{1}{a \cos \phi}\left(\frac{\partial v}{\partial \lambda}-\frac{\partial}{\partial \phi}(u \cos \phi)\right)\right)
$$

The HPE planetary vorticity thus consists solely of the vertical component $2 \Omega \mathbf{k} \sin \phi$ of the planetary vorticity $2 \Omega(\mathbf{j} \cos \phi+\mathbf{k} \sin \phi)$, and the HPE relative vorticity contains no contribution from the vertical velocity $w$. These features are what one would hope for, given (i) the shallow atmosphere aspects of the HPEs, (ii) the absence of terms in $\Omega \cos \phi$ from the zonal and vertical component equations (3.8) and (3.10), and (iii) the neglect of the term $D w / D t$. The HPE potential vorticity $\left(\alpha \mathbf{Z}_{H P E} \cdot \nabla_{a} \theta\right)$, as it appears in $(3.20)$, is of the form obtained by making similar approximations and omissions of terms in the NHD expression for potential vorticity $\alpha \mathbf{Z} \cdot \nabla \theta$ (see (2.9) and (3.2) - (3.7)). However, it is not obvious that the HPEs, as defined here in spherical polar, shallow atmosphere form, themselves actually do imply the potential vorticity law (3.20). A direct proof is given in Appendix B, and the issue is discussed further in Appendix $\mathrm{C}$ (see also section 5).

In terms of $\mathbf{v}$ and $\mathbf{Z}_{H P E}$, the horizontal components (3.8) and (3.9) may be written as

$$
\frac{\partial \mathbf{v}}{\partial t}=-\nabla_{a}\left(\frac{1}{2} \mathbf{v}^{2}\right)-\mathbf{Z}_{H P E} \times \mathbf{v}-w \frac{\partial \mathbf{v}}{\partial z}-\alpha \nabla_{a h} p+\mathbf{G}_{h}
$$

in which $\nabla_{a h}$ is the horizontal part of $\nabla_{a}$ (see (3.21)). In the HPEs, the true Coriolis force $-2 \Omega \times \mathbf{u}$, which acts in planes parallel to the Earth's equatorial plane, is replaced by $-2 \Omega \sin \phi \mathbf{k} \times \mathbf{v}$, which acts in local horizontal planes (see (3.10), (3.23) and (3.25)).

Along with the neglect of the height variation of the planetary angular momentum, the changes of both the direction and magnitude of the Coriolis force are disconcerting features of the HPEs. Nevertheless, by virtue of their conservation properties (3.18) - (3.20) and 
the Lagrange form of their momentum component equations, the HPEs satisfy our criteria for consistent formulation. Indeed, they set a standard against which any more accurate approximate model must be judged. This standard can be achieved by two more accurate models in addition to the NHD equations. Making the shallow-atmosphere approximation but not the hydrostatic approximation gives the non-hydrostatic shallow atmosphere (NHS) model; see section 3(c). Neglecting $D w / D t$ in the vertical component of the momentum equation but not making the shallow atmosphere approximation gives the quasi-hydrostatic equations (QHEs); see section 3(d). The four consistent models (NHD, NHS, QHE and HPE) and the relationships between them are represented diagrammatically in Figure 4. Table 1 summarises the approximations made in the four models, specifies the relevant forms of the differential operators and gives the angular momentum, energy and potential vorticity quantities that feature in the conservation laws.

\section{(c) The non-hydrostatic, shallow (NHS) model}

The non-hydrostatic, shallow atmosphere (NHS) model differs from the HPEs only as regards the vertical momentum balance: the term $D w / D t$ is included - as $D_{a} w / D t$ - and a non-zero component $G_{z}$ of $\mathbf{G}$ is allowed. The NHS equations are (3.8), (3.9), (3.11), (3.12), (3.15), (3.16) and

$$
\frac{D_{a} w}{D t}+g+\alpha \frac{\partial p}{\partial z}=G_{z}
$$

The shallow atmosphere approximation is made, and vertically propagating acoustic modes are present because of the retention of $D_{a} w / D t$ in (3.26). As outlined in Appendix A, the NHS momentum components (3.8), (3.9) and (3.26) may be derived from Lagrange's equations (2.10) by setting $D / D t \rightarrow D_{a} / D t$ and re-defining $T$ and $M$ (see (2.11), (2.14), $(2.15))$ as

$$
\begin{gathered}
T_{N H S} \equiv \frac{1}{2}\left[(\dot{\lambda})^{2} a^{2} \cos ^{2} \phi+(\dot{\phi})^{2} a^{2}+(\dot{z})^{2}\right], \\
M_{N H S} \equiv M_{H P E} \equiv-\dot{\lambda} \Omega a^{2} \cos ^{2} \phi .
\end{gathered}
$$

The contribution of the vertical velocity to the kinetic energy is here retained, but the shallow atmosphere approximation is applied in the same way as for the HPEs. Equivalent results were derived by Müller (1989). The NHS momentum equations were derived by Phillips (1973) (see his p.10) by setting $r=a$ in the metric factors (see Appendix A) appearing in a vector form of the NHD momentum equation.

The axial angular momentum principle of the NHS model is the same as that of the HPEs (see (3.18)). Spatial variation of $g$ is not allowed. The energy conservation law is

$$
\rho \frac{D_{a}}{D t}\left(\frac{1}{2} \mathbf{u}^{2}+g z+c_{v} T\right)+\nabla_{a} \cdot(p \mathbf{u})=\rho(Q+\mathbf{u} \cdot \mathbf{G})
$$

which is the same as the HPE form (3.19) except for the contribution of vertical motion to the kinetic energy $\left(\mathbf{u}^{2}=\mathbf{v}^{2}+w^{2}\right)$ and the rate of working by $\mathbf{G}$. The potential vorticity principle is (see Appendices B and C):

$$
\rho \frac{D_{a}}{D t}\left(\alpha \mathbf{Z}_{N H S} \cdot \nabla_{a} \theta\right)=\nabla_{a} \cdot\left(\mathbf{Z}_{N H S} \frac{D_{a} \theta}{D t}+\theta \nabla_{a} \times \mathbf{G}\right) .
$$


Here

$$
\mathbf{Z}_{N H S}=\nabla_{a} \times \mathbf{u}+2 \Omega \sin \phi \mathbf{k}=\nabla_{a} \times(\mathbf{u}+\mathbf{i} \Omega a \cos \phi),
$$

and $\nabla_{a} \times \mathbf{u}$ is the full shallow atmosphere relative vorticity, defined according to (3.22):

$$
\nabla_{a} \times \mathbf{u} \equiv\left(\frac{1}{a} \frac{\partial w}{\partial \phi}-\frac{\partial v}{\partial z}, \frac{\partial u}{\partial z}-\frac{1}{a \cos \phi} \frac{\partial w}{\partial \lambda}, \frac{1}{a \cos \phi}\left(\frac{\partial v}{\partial \lambda}-\frac{\partial}{\partial \phi}(u \cos \phi)\right)\right)
$$

The NHS momentum component equations (3.8), (3.9) and (3.26) may be combined into the vector form

$$
\frac{\partial \mathbf{u}}{\partial t}=-\nabla_{a}\left(\frac{1}{2} \mathbf{u}^{2}\right)-\mathbf{Z}_{N H S} \times \mathbf{u}-g \mathbf{k}-\alpha \nabla_{a} p+\mathbf{G} .
$$

The NHS equations have been used by, for example, Tanguay et al. (1990), Semazzi et al. (1995) and Yeh et al. (2002). They are currently available as an option in the dynamical core of the local and global weather forecasting models run by the Meteorological Service of Canada, but are not yet used operationally (Côté, private communication 2003).

\section{(d) The quasi-hydrostatic equations (QHEs)}

The QHEs differ from the NHD model only in the neglect of $D w / D t$ and $G_{r}$ from the radial component equation (2.19). The QHEs are (2.6), (2.7), (2.16) - (2.18), (2.20) and

$$
g+\alpha \frac{\partial p}{\partial r}-\frac{u^{2}}{r}-\frac{v^{2}}{r}-2 \Omega u \cos \phi=0
$$

The neglect of $D w / D t$ has the effect of removing vertically propagating acoustic modes, and puts the vertical momentum balance into a diagnostic form that differs (slightly in quantitative terrestrial terms) from the simple hydrostatic relation (3.10) - hence the designation quasi-hydrostatic. The shallow atmosphere approximation is not made. As noted in Appendix A, the QHE momentum components (2.17), (2.18) and (3.34) may be obtained from (2.10) by re-defining $T$ in the Lagrangian (2.11) as

$$
T_{Q H E} \equiv \frac{1}{2}\left[(\dot{\lambda})^{2} r^{2} \cos ^{2} \phi+(\dot{\phi})^{2} r^{2}\right]
$$

(cf. (2.14)) whilst leaving $M$ unchanged, i.e. $M_{Q H E} \equiv M$ (see (2.15)). This amounts to neglecting the explicit contribution of $w$ to the kinetic energy, but making no other change. Roulstone \& Brice (1995) gave a variational derivation of the full QHEs, including the continuity equation, and demonstrated the Poisson bracket structure of the entire model. We discuss this more comprehensive approach in section 5 .

The conservation properties of the QHEs have been discussed by White \& Bromley (1995). The angular momentum principle is the same as that of the NHD model (Eq (3.1)) because the zonal component equation (2.17) and the kinematic relations (2.16) are the same (with the same definition of the material derivative $D / D t$, i.e. (2.20)). The energy principle of the QHEs, also readily derived, is

$$
\rho \frac{D}{D t}\left(\frac{1}{2} \mathbf{v}^{2}+\Phi_{A}+c_{v} T\right)+\nabla \cdot(p \mathbf{u})=\rho(Q+\mathbf{v} \cdot \mathbf{G})
$$


This differs from the NHD form (2.8) only in the appearance of the horizontal flow $\mathbf{v} \equiv$ $(u, v, 0)$ rather than the $3 \mathrm{D}$ flow $\mathbf{u} \equiv(u, v, w)$ in the kinetic energy and in the term representing the rate-of-working by external forces. The material derivative $D / D t$ and divergence $\nabla \cdot()$ are the deep-atmosphere forms defined by (2.20) and (3.3) respectively.

The potential vorticity conservation principle of the QHEs is similar to the NHD form (2.9), but the definitions (3.5) - (3.7) of the components of absolute vorticity $\mathbf{Z}$ are modified by the omission of all terms in $w$; in vector terms, $\mathbf{Z} \rightarrow \mathbf{Z}_{Q H E} \equiv 2 \boldsymbol{\Omega}+\nabla \times \mathbf{v}$, i.e. $\mathbf{v}$ replaces $\mathbf{u}$ in the definition of relative vorticity. The principle was established for frictionless, adiabatic flow by White \& Bromley (1995), and their analysis is readily extended to the general case.

Restrictions on the spatial variations of apparent gravity are as for the NHD model: latitude variation of $g$ is not allowed, but vertical variation (as $1 / r^{2}$ ) should be included.

Until 2002, the dynamical core of the Met Office's Unified Model was a similar formulation to the QHEs, but expressed in a pressure-based, terrain-following coordinate. See section 4(b) of White \& Bromley (1995), and section 4, below, for discussion.

\section{Discussion}

The methods used in Appendices B and $\mathrm{C}$ to establish the PV conservation properties of the HPE and NHS models can be applied to other shallow atmosphere formulations. One such formulation, which we call the $r=a$ model, retains the $\cos \phi$ Coriolis terms and the spherical metric terms but sets $r=a$ wherever it occurs in undifferentiated form in the NHD equations; it is briefly and transitorily discussed in the textbooks of Gill (1982) (see p93), Holton (1992) (p37) and Dutton (1995) (p233). The PV conservation principle of the $r=a$ model, noted here in Appendix C (see (C.13)), contains terms that have no analogue in the unapproximated case. The model also lacks an acceptable axial angular momentum principle (Phillips (1968), Müller (1989)). Arguments from Lagrange's equations lead to similar conclusions: only the HPE, QHE, NHS and NHD models result from the treatment described in Appendix A.

Another strictly inconsistent model is obtained when the vertical component equation (3.34) of either deep atmosphere model (NHD, QHE) is replaced by the classical hydrostatic equation (3.10), but no other change is made.

A key aspect of the analysis so far presented is the use of height as vertical coordinate. The desirability of using other vertical coordinates in certain cases becomes clear when solution methods for the various consistent approximations in height-coordinates are considered. Since the NHD and NHS models both have prognostic equations for the vertical velocity $w$ (as well as for the horizontal velocity components) their time integration is in principle straightforward. (We leave aside the treatment of acoustic modes in the NHD and NHS models, which are in practice accommodated by the use of semi-implicit schemes and the solution of a Helmholtz equation at each timestep; see Staniforth (2001).) In contrast, the HPE and QHE models do not have a prognostic equation for $w$, so $w$ must be determined in some other way in order to carry a time integration forward.

If height is used as vertical coordinate, the procedure for determining $w$ in the HPEs is to solve a diagnostic ordinary differential equation (o.d.e.) known as Richardson's equation. See Richardson (1922), Kasahara (1974) and White (2002). It is more convenient in many 
ways to use pressure (or $\sigma=$ surface-normalised pressure) as vertical coordinate. As is well known, transformation of the HPEs to pressure coordinates leads to tractable equations without further approximation, the corresponding vertical velocity being determined from the continuity equation, which is isomorphic to an incompressible fluid form.

In the QHEs, the counterpart of Richardson's equation is much more complicated (although still a linear o.d.e. for $w$; see White (1999)). The Met Office's implementation of the QHEs in the dynamical core of its Unified Model (until 2002) depended on: (i) use of a pressure-based vertical cordinate; (ii) use of a pseudo-radius $r_{s}$ that varies only with pressure; and (iii) neglect of some very small terms. This approach was taken in order to retain good conservation properties, to eliminate acoustic modes, and to ease development from pre-existing HPE models. It is now clear that the approach was crucial in enabling the vertical velocity to be found by a continuity method (as described by White \& Bromley (1995)) rather than by the more complicated Richardson's equation method. The continuity equation remained in its convenient HPE form (as in the non-hydrostatic pressure coordinate model of Miller \& Pearce (1974), which was the first of its type).

The neglect of some very small terms in these pressure-based coordinate equations corresponds to a further degree of approximation, albeit minor, as compared with the height coordinate forms discussed in previous sections. A hierarchy of consistent, acousticallyfiltered models in pressure-based coordinates exists corresponding to the height-coordinate NHD, QHE, NHS and HPE models as we have described them, but the NHD, QHE and NHS members of that hierarchy involve further minor approximation. These approximations are mainly related to acoustic filtering in the case of the NHD and NHS pressure-based coordinate analogues. (The HPEs are of course precisely equivalent in their implemented height-coordinate and pressure-coordinate forms.) In current practice at the Met Office, the issue is circumvented by the use of the height coordinate NHD equations (after exact transformation to a terrain-following coordinate) as the dynamical core of global and limited area models; see Staniforth (2001) and Davies et al. (2004).

We also note that pressure coordinate formulations that are not acoustically filtered may be constructed by using a mass-based vertical coordinate. A compact form of the continuity equation results in this case without approximations being made; see Laprise (1992) and - for the deep-atmosphere generalization - Wood \& Staniforth (2003). Upper boundary conditions and conservation issues are considered by Staniforth et al. (2003).

\section{Conclusions}

In this paper we have identified a quartet of consistent models within the spherical geopotential approximation. These models correspond to whether approximations of hydrostatic (or quasi-hydrostatic) and shallow atmosphere type are or are not made. Consistent formulation has been regarded in terms of conservation properties for energy, angular momentum and potential vorticity, and of momentum component equations having the form of Lagrange's equations (2.10). We have also considered how the spherical geopotential and shallow atmosphere approximations place restrictions on representing the spatial variation of gravity. Each of the four consistent models has been used in numerical weather prediction and climate simulation, the most widely encountered being the hydrostatic primitive equations 
(HPEs). The HPEs are the only member of the quartet that involves hydrostatic balance in the classical form (3.10). The main features of the four consistent models are summarised in Figure 4 and Table 1.

A promising area for future study is the relationship between the treatment from Lagrange's equations (following Müller (1989)) that we have used in this paper to derive momentum component equations, and the full variational method of derivation. In the latter, one obtains the entire set of approximate equations either from an approximate version of Hamilton's principle, or demonstrates the "Poisson bracket" structure of the entire set. Methods vary in complexity according to which approach is used, although they are in the end equivalent; see Salmon (1988), Salmon (1998) and Shepherd (1990) for helpful discussions. The pressure gradient force results from a potential energy expressed in terms of particle labels, conservation properties follow from symmetries of the Hamiltonian, and their preservation under approximation is assured so long as those symmetries are maintained. A distinction is clear between approximations of the functional form of the Hamiltonian, and geometric approximations to the space in which the flow evolution occurs; see Roulstone \& Brice (1995). Müller's method is more direct than the variational methods, but - because the pressure gradient force is treated as a given field - it delivers only the components of the momentum equation, not the continuity and thermodynamic equations. The method leaves conservation properties unaddressed, but we have verified after derivation that each model of the quartet preserves conservation principles for angular momentum, energy and potential vorticity. Via the particle re-labelling symmetry of the Hamiltonian, variational methods would very probably give more direct demonstrations of potential vorticity conservation than those presented here.

Finally, we would reiterate that experience with "deep" models has suggested that they give very similar results to "shallow" models under currently prevailing terrestrial conditions [R. A. Bromley, private communication, T. Davies, private communication]. The value of deep models lies in their comprehensiveness. They apply to all planetary atmospheres for which the spherical geopotential approximation is valid, as well as to the terrestrial atmosphere even under conditions of much weaker static stability than those currently prevailing. One also feels more confident in presenting results from a deep model because the account does not have to be preceded by justification or apology for having omitted those Coriolis terms that vary as the cosine of latitude.

\section{Ackowledgements}

We thank Professor John Dutton, Professor Rick Salmon and an anonymous reviewer for their careful readings of an earlier version of this paper, and for their helpful comments. We are grateful also to Dr Mike Cullen, Dr Terry Davies and Dr Nigel Wood for useful discussions. 


\section{References}

Barnes, R. T. H., Hide, R., White, A. A. \& Wilson, C. A. 1983 , Atmospheric angular momentum fluctuations, length of day changes and polar motion, Proc. R. Soc. Lond. A 387, 31-73.

Batchelor, G. K. 1967 , An introduction to fluid dynamics, 1st edn, Cambridge University Press, Cambridge.

Bretherton, F. P. 1964, Low frequency oscillations trapped near the equator, Tellus 16, 181185.

Colin de Verdière, A. \& Schopp, R. 1994, Flows in a rotating spherical shell: the equatorial case, J. Fluid Mech. 276, 233-260.

Cullen, M. J. P. 1993 , The unified forecast/climate model, Meteorol. Mag. 122, 81-94.

Daley, R. 1988 , The normal modes of the spherical non-hydrostatic equations with applications to the filtering of acoustic modes, Tellus 40A, 96-106.

Davies, T., Cullen, M. J. P., Malcolm, A. J., Mawson, M. H., Staniforth, A., White, A. A. \& Wood, N. 2004 , A new dynamical core for the Met Office's global and regional modelling of the atmosphere, Q. J. R. Meteorol. Soc.,. (to appear).

Draghici, I. 1989 , The hypothesis of a marginally shallow atmosphere, Meteorol. Hydrol. 19, $13-27$.

Dutton, J. A. 1995 , Dynamics of atmospheric motion, 2nd edn, Dover.

Eckart, C. 1960 , The hydrodynamics of oceans and atmospheres, Pergamon Press, London.

Egger, J. 1999 , Inertial oscillations revisited, J. Atmos. Sci. 56, 2951-2954.

Gates, W. L. 2004 , Derivation of the equations of atmospheric motion in oblate spheroidal coordinates, J. Atmos. Sci. 61, 2478-2487.

Gill, A. 1982 , Atmosphere-ocean dynamics, Academic Press, London.

Goldstein, H. 1980 , Classical mechanics, 2nd edn, Addison-Wesley, London.

Holton, J. R. 1992 , An introduction to dynamic meteorology, 3rd edn, Academic Press, New York.

Kasahara, A. 1974 , Various vertical coordinate systems used for numerical weather prediction, Mon. Wea. Rev. 102, 509-522.

Kasahara, A. 2003a, On nonhydrostatic atmospheric models with inclusion of the horizontal component of the earth's angular velocity, J. Met. Soc. Japan 81, 935-950.

Kasahara, A. 2003b, The roles of the horizontal component of the earth's angular velocity in nonhydrostatic linear models, J. Atmos. Sci. 60, 1085-1095. 
Landau, L. D. \& Lifshitz, E. M. 1976 , Mechanics, 3rd edn, Elsevier, Oxford.

Laprise, R. 1992 , The Euler equations of motion with hydrostatic pressure as an independent variable, Mon. Wea. Rev. 120, 197-207.

Lorenz, E. N. 1967 , The nature and theory of the general circulation of the atmosphere, W.M.O., Geneva.

Marshall, J., Hill, C., Perelman, L. \& Adcroft, A. 1997 , Hydrostatic, quasi-hydrostatic, and non-hydrostatic ocean modeling, J. Geophys. Res. 102, 5733-5752.

Miller, M. J. \& Pearce, R. P. 1974, A three-dimensional primitive equation model of cumulonimbus convection, Q. J. R. Meteorol. Soc. 100, 133-154.

Müller, R. 1989 , A note on the relation between the 'traditional approximation' and the metric of the primitive equations, Tellus 41A, 175-178.

Newton, C. W. 1971, Global angular momentum balance: Earth torques and atmospheric fluxes, J. Atmos. Sci. 28, 1329-1341.

Norbury, J. \& Roulstone, I. 2002 , Large-scale atmosphere-ocean dynamics I and II, Cambridge University Press, Cambridge.

Oates, G. C. 1974 , Vector analysis, in C. E. Pearson, ed., 'Handbook of applied mathematics', Van Nostrand Reinhold, New York, pp. 127-178.

Pedlosky, J. 1987 , Geophysical fluid dynamics, 2nd edn, Springer-Verlag, New York.

Phillips, N. A. 1968 , Reply to 'Comments on Phillips' proposed simplification of the equations of motion for a shallow rotating atmosphere' by G. Veronis, J. Atmos. Sci. 25, 1155-1157.

Phillips, N. A. 1973 , Principles of large-scale numerical weather prediction, in Dynamic Meteorology (ed. P. Morel) Reidel, Dordrecht, pp. 1-96.

Phillips, N. A. 1990 , Dispersion processes in large-scale weather prediction, World Meteorological Organization Report No. 700, Geneva.

Richardson, L. F. 1922 , Weather prediction by numerical process, Cambridge University Press, Cambridge.

Roulstone, I. S. \& Brice, I. S. J. 1995 , On the Hamiltonian formulation of the quasihydrostatic equations, Q. J. R. Meteorol. Soc. 121, 927-936.

Salmon, R. 1988 , Hamiltonian fluid mechanics, Ann. Rev. Fluid Mech. 20, 225-256.

Salmon, R. 1998 , Lectures on geophysical fluid dynamics, Oxford University Press, Oxford.

Semazzi, F. H. M., Qian, J. H. \& Scroggs, J. S. 1995 , A global nonhydrostatic semilagrangian atmospheric model without orography, Mon. Wea. Rev. 123, 2534-2550. 
Shepherd, T. G. 1990 , Symmetries, conservation laws and hamiltonian structure in geophysical fluid dynamics, Advances in geophysics 32, 287-338.

Sivadière, J. 1983 , On the analogy between inertial and electromagnetic forces, Eur. J. Phys. 4, 162-164.

Staniforth, A. 2001 , Developing efficient unified nonhydrostatic models, in A. Spekat, ed., 'Proceedings of Symposium on 50th Anniversary of NWP, Potsdam, Germany, 9-10 March 2000', Deutsche Meteorologische Gesellschaft e.V., Berlin, pp. 185-200, 255 pp.

Staniforth, A., Wood, N. \& Girard, C. 2003 , Energy and energy-like invariants for deep non-hydrostatic atmospheres, Q. J. R. Meteorol. Soc. 129, 3495-3499.

Tanguay, M., Robert, A. \& Laprise, R. 1990 , A semi-implicit semi-Lagrangian fully compressible regional forecast model, Mon. Wea. Rev. 118, 1970-1980.

Thuburn, J., Wood, N. \& Staniforth, A. 2002a, Normal modes of deep atmospheres. I: spherical geometry, Q. J. R. Meteorol. Soc. 128, 1771-1792.

Thuburn, J., Wood, N. \& Staniforth, A. 2002b , Normal modes of deep atmospheres. II: $f-F$-plane geometry, Q. J. R. Meteorol. Soc. 128, 1793-1806.

Tomita, H. \& Satoh, M. 2004 , A new dynamical framework of nonhydrostatic global model using the icosahedral grid, Fluid Dynamics Research 34, 357-400.

White, A. A. 1999 , 'Hydrostatic and quasi-hydrostatic versions of the basic 'New Dynamics' equations'. (Available from National Meteorological Library and Archive, Met Office, Exeter, Devon, EX1 3PB, U.K. Abstract available via www.metoffice.com/research/nwp/publications/papers/technical_reports/index.html).

White, A. A. 2002, A view of the equations of meteorological dynamics and various approximations, in J. Norbury \& I. Roulstone, eds, 'Large-scale atmosphere-ocean dynamics I', Cambridge University Press, Cambridge, pp. 1-100.

White, A. A. 2003 , Primitive equations, in J. R. Holton et al., eds, 'Encyclopedia of atmospheric science', Academic Press, New York, pp. 694-702.

White, A. A. \& Bromley, R. A. 1995 , Dynamically consistent, quasi-hydrostatic equations for global models with a complete representation of the Coriolis force, Q. J. R. Meteorol. Soc. 121, 399-418.

Wood, N. \& Staniforth, A. 2003 , The deep-atmosphere Euler equations with a mass-based vertical coordinate, Q. J. R. Meteorol. Soc. 129, 1289-1300.

Yeh, K.-S., Côté, J., Gravel, S., Méthot, A., Patoine, A., Roch, M. \& Staniforth, A. 2002 , The CMC-MRB Global Environmental Multiscale (GEM) model. Part III: Nonhydrostatic formulation, Mon. Wea. Rev. 130, 339-356.

Zdunkowski, W. \& Bott, A. 2003 , Dynamics of the atmosphere: a course in theoretical meteorology, 1st edn, Cambridge University Press, Cambridge. 


\section{APPENDIX A}

\section{Lagrange's form of the component momentum equations in a rotating system} and their approximation

The treatment given by Müller (1989) (following K. Hinkelmann) has recently been presented in more detail by Zdunkowski \& Bott (2003); see their chapter 18. Both studies use velocity in the absolute frame as starting point and both work in general coordinates. Here we limit attention to orthogonal coordinate systems, work in the rotating frame throughout, and offer a free-standing treatment tailored to our context. The incorporation of the Lorentz force into the Lagrange framework is described in analytical dynamics texts such as Goldstein (1980). The Coriolis force $-2 \boldsymbol{\Omega} \times \mathbf{u}$ is easier to handle than the Lorentz force $-\mathbf{B} \times \mathbf{u}$ because spatial variations of $\boldsymbol{\Omega}$ do not have to be allowed for. [B is the magnetic field; $\mathbf{B} \equiv \nabla \times \mathbf{A}$, where $\mathbf{A}$ is a vector potential. $2 \boldsymbol{\Omega}=\nabla \times(\boldsymbol{\Omega} \times \mathbf{r})$.]

In a Cartesian system $O x_{1} x_{2} x_{3}$ rotating with the frame $\Im$, the $j$ th component $(j=1,2,3)$ of $(2.2)$ is

$$
\frac{D u_{j}}{D t}=-2(\boldsymbol{\Omega} \times \mathbf{u})_{j}-\frac{\partial \Phi_{A}}{\partial x_{j}}-\alpha \frac{\partial p}{\partial x_{j}}+G_{j},
$$

where $u_{j}=D x_{j} / D t=\dot{x}_{j}$. In terms of the specific kinetic energy, $T$, given by

$$
2 T=\sum_{j=1}^{j=3} u_{j}^{2}=\sum_{j=1}^{j=3}\left(\dot{x}_{j}\right)^{2}
$$

and the scalar field $M$ defined as

$$
M=-\mathbf{u} \cdot(\boldsymbol{\Omega} \times \mathbf{r})=-\dot{\mathbf{r}} \cdot(\boldsymbol{\Omega} \times \mathbf{r})=\mathbf{r} \cdot(\boldsymbol{\Omega} \times \dot{\mathbf{r}}),
$$

(where $\left.\mathbf{r} \equiv\left(x_{1}, x_{2}, x_{3}\right)\right)$, (A.1) can be written as

$$
\frac{D}{D t}\left(\frac{\partial T}{\partial \dot{x}_{j}}\right)=\frac{D}{D t}\left(\frac{\partial M}{\partial \dot{x}_{j}}\right)-\frac{\partial M}{\partial x_{j}}-\frac{\partial \Phi_{A}}{\partial x_{j}}-\alpha \frac{\partial p}{\partial x_{j}}+G_{j} .
$$

Since $T$ does not depend explicitly on the $x_{j}$, and the apparent gravitational potential $\Phi_{A}$ does not depend on the $\dot{x}_{j}$, (A.1) may be written compactly as

$$
\frac{D}{D t}\left(\frac{\partial L}{\partial \dot{x}_{j}}\right)-\frac{\partial L}{\partial x_{j}}=-\alpha \frac{\partial p}{\partial x_{j}}+G_{j},
$$

in which the Lagrangian $L$ function is given by

$$
L=L\left(x_{j}, \dot{x}_{j}\right)=T-M-\Phi_{A} .
$$

The quantity $M$ defined by (A.3) may be regarded as a velocity-dependent potential (which, according to Goldstein (1980), p21, has been called the Schering potential). Alternatively, $-M$ can be regarded as an additional contribution to the kinetic energy: it is the "cross term" in the absolute kinetic energy $\frac{1}{2}(\mathbf{u}+\boldsymbol{\Omega} \times \mathbf{r})^{2}$. This interpretation is appropriate to the absolute-frame treatment of Müller (1989); see also Landau \& Lifshitz (1976), p128. (The term $\frac{1}{2}(\boldsymbol{\Omega} \times \mathbf{r})^{2}$ in the absolute kinetic energy appears in (A.6) as part of $-\Phi_{A}$.) 
To transform (A.5) to the system $q_{i}=q_{i}\left(x_{j}\right)$, multiply by $\partial x_{j} / \partial q_{i}$ and sum over $j$ :

$$
\sum_{j=1}^{j=3}\left[\frac{D}{D t}\left(\frac{\partial L}{\partial \dot{x}_{j}} \frac{\partial x_{j}}{\partial q_{i}}\right)-\frac{\partial L}{\partial \dot{x}_{j}} \frac{D}{D t}\left(\frac{\partial x_{j}}{\partial q_{i}}\right)-\frac{\partial L}{\partial x_{j}} \frac{\partial x_{j}}{\partial q_{i}}\right]=-\alpha \frac{\partial p}{\partial q_{i}}+\sum_{j=1}^{j=3} G_{j} \frac{\partial x_{j}}{\partial q_{i}}
$$

From the inverse relation $x_{j}=x_{j}\left(q_{i}\right)$ it follows that $\partial x_{j} / \partial \dot{q}_{i}=0$; also,

$$
\dot{x}_{j}=\sum_{k=1}^{k=3} \frac{\partial x_{j}}{\partial q_{k}} \dot{q}_{k} \Rightarrow \frac{\partial \dot{x}_{j}}{\partial \dot{q}_{i}}=\frac{\partial x_{j}}{\partial q_{i}} \text { and } \frac{\partial \dot{x}_{j}}{\partial q_{i}}=\sum_{k=1}^{k=3} \frac{\partial^{2} x_{j}}{\partial q_{i} \partial q_{k}} \dot{q}_{k}=\frac{D}{D t}\left(\frac{\partial x_{j}}{\partial q_{i}}\right) .
$$

Hence

$$
\begin{gathered}
\frac{\partial L}{\partial \dot{q}_{i}}=\frac{\partial}{\partial \dot{q}_{i}}\left[L\left(x_{j}, \dot{x}_{j}\right)\right]=\sum_{j=1}^{j=3}\left[\frac{\partial L}{\partial x_{j}} \frac{\partial x_{j}}{\partial \dot{q}_{i}}+\frac{\partial L}{\partial \dot{x_{j}}} \frac{\partial \dot{x}_{j}}{\partial \dot{q}_{i}}\right]=\sum_{j=1}^{j=3}\left[\frac{\partial L}{\partial \dot{x}_{j}} \frac{\partial x_{j}}{\partial q_{i}}\right] \\
\frac{\partial L}{\partial q_{i}}=\frac{\partial}{\partial q_{i}}\left[L\left(x_{j}, \dot{x}_{j}\right)\right]=\sum_{j=1}^{j=3}\left[\frac{\partial L}{\partial x_{j}} \frac{\partial x_{j}}{\partial q_{i}}+\frac{\partial L}{\partial \dot{x}_{j}} \frac{\partial \dot{x}_{j}}{\partial q_{i}}\right]=\sum_{j=1}^{j=3}\left[\frac{\partial L}{\partial x_{j}} \frac{\partial x_{j}}{\partial q_{i}}+\frac{\partial L}{\partial \dot{x}_{j}} \frac{D}{D t}\left(\frac{\partial x_{j}}{\partial q_{i}}\right)\right],
\end{gathered}
$$

and (A.7) becomes

$$
\frac{D}{D t}\left(\frac{\partial L}{\partial \dot{q}_{i}}\right)-\frac{\partial L}{\partial q_{i}}=-\alpha \frac{\partial p}{\partial q_{i}}+\sum_{j=1}^{j=3} G_{j} \frac{\partial x_{j}}{\partial q_{i}}
$$

The tortuous path leading from (A.5) to (A.11) is generically standard (see Goldstein (1980), p19) as indeed is (A.11) . We have described it for the sake of clarity and completeness.

In terms of the (non-negative) metric factors $h_{i}$ defined by

$$
h_{i}^{2}=\sum_{j=1}^{j=3}\left(\frac{\partial x_{j}}{\partial q_{i}}\right)^{2}
$$

and mutually orthogonal unit vectors $\widehat{\mathbf{q}}_{i}$ in the directions $q_{i}$ we have

$$
\mathbf{u}=\sum_{i=1}^{i=3} h_{i} \dot{q}_{i} \widehat{\mathbf{q}}_{i} \Rightarrow \mathbf{u}^{2}=2 T=\sum_{i=1}^{i=3} h_{i}^{2}\left(\dot{q}_{i}\right)^{2}
$$

$M$ can also be written in terms of the $q_{i}$ and $\dot{q}_{i}$, but the result is not informative in the general case. $\Phi_{A}=\Phi_{A}\left(q_{i}\right)$ is in principle readily obtainable from $\Phi_{A}=\Phi_{A}\left(x_{j}\right)$, given $x_{j}=x_{j}\left(q_{i}\right)$.

Upon division by the metric factor $h_{i}$, the sum on the right-hand side of (A.11) becomes simply the component of vector $\mathbf{G}$ in the direction of the coordinate $q_{i}$, which we denote (with tolerable ambiguity) $G_{i}$. Hence (A.11) can be written

$$
\frac{D}{D t}\left(\frac{\partial L}{\partial \dot{q}_{i}}\right)-\frac{\partial L}{\partial q_{i}}=-\alpha \frac{\partial p}{\partial q_{i}}+h_{i} G_{i},
$$

in which there is no summation over the repeated suffix $i$ in the final product term.

In the spherical geopotential approximation, choose spherical polar coordinates $q_{1}=\lambda=$ longitude, $q_{2}=\phi=$ latitude, $q_{3}=r=$ radial distance, and require $\Phi_{A}=\Phi_{A}(r)$. Use of

$$
x_{1}=r \cos \phi \sin \lambda, x_{2}=r \cos \phi \cos \lambda \text { and } x_{3}=r \sin \phi
$$


in (A.12) gives, as expected,

$$
h_{1}=h_{\lambda}=r \cos \phi, h_{2}=h_{\phi}=r ; \text { and } h_{3}=h_{r}=1 .
$$

Eqs. (A.13) and (A.3) now deliver (2.14) and (2.15) as

$$
2 T \equiv \mathbf{u}^{2}=(\dot{\lambda})^{2} r^{2} \cos ^{2} \phi+(\dot{\phi})^{2} r^{2}+(\dot{r})^{2} \quad \text { and } \quad M=-\dot{\lambda} \Omega r^{2} \cos ^{2} \phi
$$

With Eqs. (A.17) as they stand, and with $\Phi_{A}=\Phi_{A}(r)$, we find from (A.6):

$$
\begin{gathered}
\frac{\partial L}{\partial \dot{\lambda}}=(\dot{\lambda}+\Omega) r^{2} \cos ^{2} \phi \text { and } \frac{\partial L}{\partial \lambda}=0 \\
\frac{\partial L}{\partial \dot{\phi}}=\dot{\phi} r^{2} \text { and } \frac{\partial L}{\partial \phi}=-\dot{\lambda}(\dot{\lambda}+2 \Omega) r^{2} \sin \phi \cos \phi \\
\frac{\partial L}{\partial \dot{r}}=\dot{r} \text { and } \frac{\partial L}{\partial r}=\dot{\lambda}(\dot{\lambda}+2 \Omega) r^{2} \cos ^{2} \phi+(\dot{\phi})^{2} r-\frac{\partial \Phi_{A}}{\partial r} .
\end{gathered}
$$

Use of (A.18) in (A.14) with $q_{i}=q_{1}=\lambda$ leads to the zonal component equation (2.17) of the NHD equations. Similarly, use of (A.19) in (A.14) with $q_{i}=q_{2}=\phi$ leads to (2.18). Finally, use of (A.20) in (A.14) with $q_{i}=q_{3}=r$ gives (2.19). Repeating this procedure, but omitting the contribution of $w=\dot{r}$ to the kinetic energy, i.e. with

$$
2 T \rightarrow 2 T_{Q H E} \equiv \mathbf{v}^{2}=(\dot{\lambda})^{2} r^{2} \cos ^{2} \phi+(\dot{\phi})^{2} r^{2},
$$

is easily seen to deliver the QHE momentum components (2.17), (2.18) and (3.34).

Making the shallow atmosphere approximation in the form $r \rightarrow a$ in (A.17) gives

$$
2 T \rightarrow 2 T_{N H S}=(\dot{\lambda})^{2} a^{2} \cos ^{2} \phi+(\dot{\phi})^{2} a^{2}+(\dot{r})^{2} \text { and } M \rightarrow M_{N H S}=-\dot{\lambda} \Omega a^{2} \cos ^{2} \phi .
$$

In place of (A.18) - (A.20), with $L \rightarrow T_{N H S}-M_{N H S}-\Phi_{A}(r)$ we find

$$
\begin{gathered}
\frac{\partial L}{\partial \dot{\lambda}}=(\dot{\lambda}+\Omega) a^{2} \cos ^{2} \phi \text { and } \frac{\partial L}{\partial \lambda}=0 ; \\
\frac{\partial L}{\partial \dot{\phi}}=\dot{\phi} a^{2} \text { and } \frac{\partial L}{\partial \phi}=-\dot{\lambda}(\dot{\lambda}+2 \Omega) a^{2} \sin \phi \cos \phi ; \\
\frac{\partial L}{\partial \dot{r}}=\dot{r} \text { and } \frac{\partial L}{\partial r}=-\frac{d \Phi_{A}}{d r} .
\end{gathered}
$$

The NHS equations can be obtained by repeating the procedure by which the NHD equations were earlier derived from (A.14) by use of (A.18) - (A.20), but with $D / D t$ replaced by $D_{a} / D t$ (which corresponds to setting $r=a$ in (2.20). Finally, the HPEs result when the contribution of $w=\dot{r}$ to the kinetic energy is neglected in (A.22), i.e. when

$$
2 T_{N H S} \rightarrow 2 T_{H P E}=(\dot{\lambda})^{2} a^{2} \cos ^{2} \phi+(\dot{\phi})^{2} a^{2} .
$$

In addition to the simplifying restriction to orthogonal coordinate systems, this treatment differs from that of Müller (1989) and Zdunkowski \& Bott (2003) as regards the partition between kinetic and potential energy; we have worked throughout in the rotating frame. Setting $r=a$ in (A.17) and (2.20) is the metric simplification of Müller (1989) and Zdunkowski $\&$ Bott (2003), and leads to the NHS equations. An essentially new element of the present treatment is its inclusion of the QHE and HPE models in the picture by re-defining the specific kinetic energy so as to exclude the contribution of the vertical velocity. An anonymous reviewer has observed that this re-definition is a degeneration of the velocity metric. 


\section{APPENDIX B}

\section{Direct proof of the HPE PV equation (3.20)}

Introduce horizontal coordinates $\widehat{x}, \widehat{y}$ and weighted velocity components $\widehat{u}, \widehat{v}$ as

$$
\widehat{x}=\lambda a \cos ^{2} \phi, \widehat{y}=a \sin \phi \quad \text { and } \quad \widehat{u}=u \cos \phi, \widehat{v}=v \cos \phi
$$

and set

$$
\widehat{\nabla}=(\partial / \partial \widehat{x}, \partial / \partial \widehat{y}, \partial / \partial z), \quad \widehat{\mathbf{u}}=(\widehat{u}, \widehat{v}, w) .
$$

From (3.11), (B.1) and (B.2) it follows that, for any once-differentiable field $\beta$,

$$
\frac{D_{a} \beta}{D t}=\left(\frac{\partial}{\partial t}+\widehat{\mathbf{u}} \cdot \widehat{\nabla}\right) \beta
$$

The operators $\partial / \partial \widehat{x}$ (at constant $\phi$ ) and $\partial / \partial \widehat{y}$ (at constant $\lambda$ ) do not commute. With $Y=\sin \phi=\widehat{y} / a$ :

$$
\frac{\partial}{\partial \widehat{x}} \frac{\partial}{\partial \widehat{y}}-\frac{\partial}{\partial \widehat{y}} \frac{\partial}{\partial \widehat{x}}=-\frac{2 Y}{a\left(1-Y^{2}\right)} \frac{\partial}{\partial \widehat{x}}
$$

But $\partial / \partial \widehat{x}$ and $\partial / \partial \widehat{y}$ each commute with $\partial / \partial z$ (which acts at constant $\phi$ and $\lambda$ ). Hence

$$
\begin{gathered}
\frac{\partial}{\partial \widehat{x}}\left(\frac{D_{a} \beta}{D t}\right)=\frac{D_{a}}{D t}\left(\frac{\partial \beta}{\partial \widehat{x}}\right)+\frac{\partial \widehat{\mathbf{u}}}{\partial \widehat{x}} \cdot \widehat{\nabla} \beta-\frac{2 Y \widehat{v}}{a\left(1-Y^{2}\right)} \frac{\partial \beta}{\partial \widehat{x}} \\
\frac{\partial}{\partial \widehat{y}}\left(\frac{D_{a} \beta}{D t}\right)=\frac{D_{a}}{D t}\left(\frac{\partial \beta}{\partial \widehat{y}}\right)+\frac{\partial \widehat{\mathbf{u}}}{\partial \widehat{y}} \cdot \widehat{\nabla} \beta+\frac{2 Y \widehat{u}}{a\left(1-Y^{2}\right)} \frac{\partial \beta}{\partial \widehat{x}} \\
\frac{\partial}{\partial z}\left(\frac{D_{a} \beta}{D t}\right)=\frac{D_{a}}{D t}\left(\frac{\partial \beta}{\partial z}\right)+\frac{\partial \widehat{\mathbf{u}}}{\partial z} \cdot \widehat{\nabla} \beta .
\end{gathered}
$$

Since $\nabla_{a} \cdot \mathbf{u}$, as defined by (3.17), transforms to $\widehat{\nabla} \cdot \widehat{\mathbf{u}}$, the continuity equation (3.16) becomes

$$
\frac{D_{a} \alpha}{D t}=\alpha \widehat{\nabla} \cdot \widehat{\mathbf{u}}
$$

The horizontal component equations (3.8) and (3.9) of the HPEs can be written

$$
\begin{gathered}
\frac{D_{a} \widehat{u}}{D t}=2 \Omega \widehat{v} Y+H_{\lambda}\left(1-Y^{2}\right)^{\frac{1}{2}} \\
\frac{D_{a} \widehat{v}}{D t}=-2 \Omega \widehat{u} Y-\frac{\left(\widehat{u}^{2}+\widehat{v}^{2}\right) Y}{a\left(1-Y^{2}\right)}+H_{\phi}\left(1-Y^{2}\right)^{\frac{1}{2}}
\end{gathered}
$$

in which $H_{\lambda}$ and $H_{\phi}$ are the zonal and meridional components of the vector $\mathbf{H}_{h}=\mathbf{G}_{h}-\alpha \nabla_{a h} p$, $\nabla_{a h}$ being the horizontal part of $\nabla_{a}$ (see (3.21)).

Define a modified HPE absolute vorticity $\widehat{\mathbf{Z}}$ in terms of $\widehat{\mathbf{v}}=(\widehat{u}, \widehat{v}, 0)$ as

$$
\widehat{\mathbf{Z}}=\left(\widehat{Z}_{1}, \widehat{Z}_{2}, \widehat{Z}_{3}\right)=\widehat{\nabla} \times \widehat{\mathbf{v}}+f \mathbf{k}=\left(-\frac{\partial \widehat{v}}{\partial z}, \frac{\partial \widehat{u}}{\partial z}, 2 \Omega Y+\frac{\partial \widehat{v}}{\partial \widehat{x}}-\frac{\partial \widehat{u}}{\partial \widehat{y}}\right) .
$$


From (B.9) - (B.11), using (B.5) - (B.7), it follows that

$$
\begin{gathered}
\frac{D_{a} \widehat{Z}_{1}}{D t}=(\widehat{\mathbf{Z}} \cdot \widehat{\nabla}) \widehat{u}-\widehat{Z}_{1} \widehat{\nabla} \cdot \widehat{\mathbf{u}}+\frac{Y}{a\left(1-Y^{2}\right)} \frac{\partial}{\partial z}\left(\widehat{u}^{2}+\widehat{v}^{2}\right)-\left(1-Y^{2}\right)^{\frac{1}{2}} \frac{\partial H_{\phi}}{\partial z}, \\
\frac{D_{a} \widehat{Z}_{2}}{D t}=(\widehat{\mathbf{Z}} \cdot \widehat{\nabla}) \widehat{v}-\widehat{Z}_{2} \widehat{\nabla} \cdot \widehat{\mathbf{u}}+\left(1-Y^{2}\right)^{\frac{1}{2}} \frac{\partial H_{\lambda}}{\partial z}, \\
\frac{D_{a} \widehat{Z}_{3}}{D t}=(\widehat{\mathbf{Z}} \cdot \widehat{\nabla}) w-\widehat{Z}_{3} \widehat{\nabla} \cdot \widehat{\mathbf{u}}+\frac{\partial}{\partial \widehat{x}}\left[H_{\phi}\left(1-Y^{2}\right)^{\frac{1}{2}}\right]-\frac{\partial}{\partial \widehat{y}}\left[H_{\lambda}\left(1-Y^{2}\right)^{\frac{1}{2}}\right] .
\end{gathered}
$$

Use of (B.8) in (B.12)-(B.14), and multiplication by components of $\widehat{\nabla} \theta$, gives

$$
\begin{aligned}
\frac{\partial \theta}{\partial \widehat{x}} \frac{D_{a}}{D t}\left(\alpha \widehat{Z}_{1}\right)= & \alpha \frac{\partial \theta}{\partial \widehat{x}}(\widehat{\mathbf{Z}} \cdot \widehat{\nabla}) \widehat{u}+\alpha \frac{\partial \theta}{\partial \widehat{x}}\left[\frac{Y}{a\left(1-Y^{2}\right)} \frac{\partial}{\partial z}\left(\widehat{u}^{2}+\widehat{v}^{2}\right)-\left(1-Y^{2}\right)^{\frac{1}{2}} \frac{\partial H_{\phi}}{\partial z}\right] \\
& \frac{\partial \theta}{\partial \widehat{y}} \frac{D_{a}}{D t}\left(\alpha \widehat{Z} \widehat{Z}_{2}\right)=\alpha \frac{\partial \theta}{\partial \widehat{y}}(\widehat{\mathbf{Z}} \cdot \widehat{\nabla}) \widehat{v}+\alpha \frac{\partial \theta}{\partial \widehat{y}}\left(1-Y^{2}\right)^{\frac{1}{2}} \frac{\partial H_{\lambda}}{\partial z} \\
\frac{\partial \theta}{\partial z} \frac{D_{a}}{D t}\left(\alpha \widehat{Z}_{3}\right)= & \alpha \frac{\partial \theta}{\partial z}(\widehat{\mathbf{Z}} \cdot \widehat{\nabla}) w+\alpha \frac{\partial \theta}{\partial z}\left\{\frac{\partial}{\partial \widehat{x}}\left[H_{\phi}\left(1-Y^{2}\right)^{\frac{1}{2}}\right]-\frac{\partial}{\partial \widehat{y}}\left[H_{\lambda}\left(1-Y^{2}\right)^{\frac{1}{2}}\right]\right\} .
\end{aligned}
$$

Put $\beta=\theta$ in (B.5)-(B.7), multiply by components of $\alpha \widehat{\mathbf{Z}}$ and note (B.11) to obtain:

$$
\begin{aligned}
\alpha \widehat{Z}_{1} \frac{D_{a}}{D t}\left(\frac{\partial \theta}{\partial \widehat{x}}\right)= & -\alpha \widehat{Z}_{1} \frac{\partial \widehat{\mathbf{u}}}{\partial \widehat{x}} \cdot \widehat{\nabla} \theta-\frac{\alpha Y}{a\left(1-Y^{2}\right)} \frac{\partial \theta}{\partial \widehat{x}} \frac{\partial}{\partial z}\left(\widehat{v}^{2}\right)+\alpha \widehat{Z}_{1} \frac{\partial}{\partial \widehat{x}}\left(\frac{D_{a} \theta}{D t}\right), \\
\alpha \widehat{Z}_{2} \frac{D_{a}}{D t}\left(\frac{\partial \theta}{\partial \widehat{y}}\right)= & -\alpha \widehat{Z}_{2} \frac{\partial \widehat{\mathbf{u}}}{\partial \widehat{y}} \cdot \widehat{\nabla} \theta-\frac{\alpha Y}{a\left(1-Y^{2}\right)} \frac{\partial \theta}{\partial \widehat{x}} \frac{\partial}{\partial z}\left(\widehat{u}^{2}\right)+\alpha \widehat{Z}_{2} \frac{\partial}{\partial \widehat{y}}\left(\frac{D_{a} \theta}{D t}\right), \\
& \alpha \widehat{Z}_{3} \frac{D_{a}}{D t}\left(\frac{\partial \theta}{\partial z}\right)=-\alpha \widehat{Z}_{3} \frac{\partial \widehat{\mathbf{u}}}{\partial z} \cdot \widehat{\nabla} \theta+\alpha \widehat{Z}_{3} \frac{\partial}{\partial z}\left(\frac{D_{a} \theta}{D t}\right) .
\end{aligned}
$$

Upon addition of (B.15)-(B.20), the six explicit scalar products on the right-hand sides cancel, as do the terms involving $\widehat{u}^{2}$ and $\widehat{v}^{2}$. The result can be written

$$
\frac{D_{a}}{D t}(\alpha \widehat{\mathbf{Z}} \cdot \widehat{\nabla} \theta)=\alpha \widehat{\mathbf{Z}} \cdot \widehat{\nabla}\left(\frac{D_{a} \theta}{D t}\right)+\alpha \widehat{\nabla} \theta \cdot \widehat{\nabla} \times\left[\left(1-Y^{2}\right)^{\frac{1}{2}} \mathbf{H}_{h}\right]
$$

In terms of $\mathbf{Z}_{H P E}$ and $\nabla_{a}$ rather than $\widehat{\mathbf{Z}}$ and $\widehat{\nabla}$ (note $(3.23)$ ), (B.21) becomes

$$
\frac{D_{a}}{D t}\left(\alpha \mathbf{Z}_{H P E} \cdot \nabla_{a} \theta\right)=\alpha \mathbf{Z}_{H P E} \cdot \nabla_{a}\left(\frac{D_{a} \theta}{D t}\right)+\alpha\left(\nabla_{a} \times \mathbf{H}_{h}\right) \cdot \nabla_{a} \theta .
$$

Since $\mathbf{H}_{h}=\mathbf{G}_{h}-\alpha \nabla_{a h} p$, and $g=$ constant in the hydrostatic relation (3.10),

$$
\nabla_{a} \times \mathbf{H}_{h}=\nabla_{a} \times \mathbf{G}_{h}-\nabla_{a} \times\left(\alpha \nabla_{a} p\right)=\nabla_{a} \times \mathbf{G}_{h}-\nabla_{a} \alpha \times \nabla_{a} p .
$$

Hence $\nabla_{a} \theta \cdot \nabla_{a} \times \mathbf{H}_{h}=\nabla_{a} \theta \cdot \nabla_{a} \times \mathbf{G}_{h}$ (because $\alpha=\alpha(p, \theta) \Rightarrow \nabla_{a} \theta \cdot\left(\nabla_{a} \alpha \times \nabla_{a} p\right)=0$ ) and (B.22) reduces to $(3.20)$. 
This direct method may be used to examine the PV conservation properties of other shallow atmosphere models. Other terms may be included in the component momentum equations by suitably re-defining $H_{\lambda}$ and $H_{\phi}$ in (B.9) and (B.10), and allowing a vertical component $H_{z}$ if necessary. New or suspected components of the appropriate absolute vorticity may be included by augmenting the components of $\widehat{\mathbf{Z}}$ (see (B.11)). The PV conservation law (3.30) of the NHS model can be derived in this way, but a method that exploits the vector form of (3.33) is quicker, given a property of vector differential identities that is discussed in Appendix C.

\section{APPENDIX C}

\section{Metric approximation and vector differential identities}

As is well known, the potential vorticity equation (2.9) may be derived from the unapproximated equations (2.1), (2.6) and (2.7) via the vorticity equation (2.5) by using various identities involving the vector differential operators grad, div and $\operatorname{curl}(\nabla, \nabla \cdot$ and $\nabla \times)$ :

$$
\begin{gathered}
2(\mathbf{A} \cdot \nabla) \mathbf{A}=\nabla\left(\mathbf{A}^{2}\right)-2 \mathbf{A} \times(\nabla \times \mathbf{A}), \\
\nabla \times(\mathbf{A} \times \mathbf{B})=\mathbf{A} \nabla \cdot \mathbf{B}+(\mathbf{B} \cdot \nabla) \mathbf{A}-\mathbf{B} \nabla \cdot \mathbf{A}-(\mathbf{A} \cdot \nabla) \mathbf{B}, \\
\nabla \times(\chi \mathbf{A})=\chi \nabla \times \mathbf{A}+(\nabla \chi) \times \mathbf{A}, \\
(\mathbf{A} \cdot \nabla) \frac{D \chi}{D t}=\mathbf{A} \cdot \frac{D}{D t}(\nabla \chi)+\nabla \chi \cdot[(\mathbf{A} \cdot \nabla) \mathbf{u}] .
\end{gathered}
$$

(See, for example, Pedlosky (1987), p38.) Here A, B and $\chi$ are suitably differentiable vector and scalar fields. In addition, some more basic properties of the operators are needed:

$$
\nabla \times \nabla \chi=0, \quad \nabla \cdot(\nabla \times \mathbf{A})=0 .
$$

The identities (C.1) - (C.5) are usually established by adopting a Cartesian representation of the vectors and using the appropriate and familiar definitions of grad, div and curl. In specific physical contexts, it is often convenient to use curvilinear orthogonal coordinate systems (cylindrical polar, spherical polar, parabolic cylinder coordinates etc.) and corresponding expressions for grad div and curl are then required. The procedure in each case is to derive exact transforms of the familiar Cartesian expressions. Given appropriate orthogonal coordinates $q_{1}, q_{2}, q_{3}$, and metric factors $h_{1}, h_{2}, h_{3}$ (as defined in Appendix A), the relevant expressions are

$$
\begin{gathered}
(\nabla \chi)_{j}=\frac{1}{h_{j}} \frac{\partial \chi}{\partial q_{j}} \\
\nabla \cdot \mathbf{A}=\frac{1}{h_{1} h_{2} h_{3}}\left[\frac{\partial}{\partial q_{1}}\left(h_{2} h_{3} A_{1}\right)+\frac{\partial}{\partial q_{2}}\left(h_{3} h_{1} A_{2}\right)+\frac{\partial}{\partial q_{3}}\left(h_{1} h_{2} A_{3}\right)\right] \\
(\nabla \times \mathbf{A})_{j}=\frac{h_{j}}{h_{1} h_{2} h_{3}} \sum_{k, l} \epsilon_{j k l} \frac{\partial}{\partial q_{k}}\left(h_{l} A_{l}\right) .
\end{gathered}
$$


In (C.8), $\epsilon_{j k l}$ is the usual cyclic permutation tensor. The corresponding expression for the $j$ th component of the advective derivative $(\mathbf{A} \cdot \nabla) \mathbf{B}$ is

$$
[(\mathbf{A} \cdot \nabla) \mathbf{B}]_{j}=\sum_{i}\left\{\frac{A_{i}}{h_{i}} \frac{\partial B_{j}}{\partial q_{i}}+\frac{B_{i}}{h_{i} h_{j}}\left(A_{j} \frac{\partial h_{j}}{\partial q_{i}}-A_{i} \frac{\partial h_{i}}{\partial q_{j}}\right)\right\} .
$$

(See Batchelor (1967), p598 and Oates (1974), p152, for derivations of (C.9).) That the identities (C.1) - (C.5) are preserved in the curvilinear orthogonal system is assured for the very reason that the transformation from the Cartesian system is exact.

The discussion so far has applied to everyday 3D space. A new perspective is attained by noting that the general orthogonal curvilinear coordinate expressions (C.6) - (C.9) satisfy (C.1) - (C.5) irrespective of the functional dependences of the metric factors $h_{1}, h_{2}, h_{3}$ on the coordinates $q_{1}, q_{2}, q_{3}$. (It is assumed that the dependences are appropriately differentiable.) To establish this result, (C.6) - (C.9) may be considered as definitions of the various differential operations on the appropriate fields, and the relations (C.1) - (C.5) verified directly. The procedure is straightforward for all cases, but for some (e.g. (C.4)) considerable labour is required. The result has important ramifications for approximation. If the functional dependences of the metric factors are changed from their usual 3D-space forms in a particular coordinate system, then a mathematical space is set up which is a distortion of 3D physical space and is non-Euclidean; but the vector differential operators defined in this mathematical space behave analogously to the usual 3D-space operators. In the case we are interested in here, the shallow spherical polar choice $h_{1}=a \cos \phi, h_{2}=a, h_{3}=1$ gives the definitions of grad, div and curl noted in section 3(c), and preservation of the identities (C.1) - (C.5) is assured so long as we retain those definitions throughout. A PV law of the form (3.30) follows: its derivation from (3.33), (3.15) and (3.16) proceeds in parallel with that for the unapproximated case. It is crucial that the NHS momentum component equations (3.8), (3.9) and (3.26) can indeed be combined into the vector form (3.33).

The argument may be extended to other conservation principles by showing that the relevant vector identities are also indifferent to the functional dependences of the metric factors on the coordinates.

Several published studies discuss the shallow atmosphere approximation and the NHS model in terms of replacing the variable radius $r$ by the constant $a$ in the metric factors; see, for example, Phillips (1973) p10 and Zdunkowski \& Bott (2003) p538. But, so far as we are aware, the result that approximating the metric factors preserves the conservation properties has not previously been stated in a meteorological context.

The PV conservation properties of other shallow atmosphere models may be examined after placing the appropriate terms in the vector momentum equation (3.33). An example is the $r=a$ model, discussed in section 4 , in which all the spherical metric and $\cos \phi$ Coriolis terms are included but $r$ is set equal to $a$ wherever it appears in undifferentiated form. The component momentum equations can be gathered into the (shallow-atmosphere) vector form

$$
\frac{\partial \mathbf{u}}{\partial t}=-\nabla_{a}\left(\frac{1}{2} \mathbf{u}^{2}\right)-\widetilde{\mathbf{Z}} \times \mathbf{u}-\frac{1}{a}(\mathbf{k} \times \mathbf{u}) \times \mathbf{u}-g \mathbf{k}-\alpha \nabla_{a} p+\mathbf{G} .
$$

Here

$$
\widetilde{\mathbf{Z}}=\nabla_{a} \times \mathbf{u}+2 \boldsymbol{\Omega}
$$


is the natural definition of absolute vorticity for this model. Forming a vorticity equation by taking $\nabla_{a} \times\left(\right.$ C.10) generates spurious terms via $\nabla_{a} \times(\mathbf{u} \times(\mathbf{k} \times \mathbf{u}))=\nabla_{a} \times\left(\mathbf{k}\left(\mathbf{v}^{2}\right)-w \mathbf{v}\right)$. A spurious term is also introduced by $\nabla_{a} \times(2 \Omega \times \mathbf{u})$ when the shallow atmosphere version of (C.2) is applied, since $\boldsymbol{\Omega}$ is divergent in shallow-atmosphere space:

$$
\nabla_{a} \cdot \Omega=\nabla_{a} \cdot(0, \Omega \cos \phi, \Omega \sin \phi)=-\frac{2 \Omega}{a} \sin \phi
$$

The only way in which this spurious effect can be removed is to neglect the horizontal component of $\boldsymbol{\Omega}$ - which amounts, of course, to the neglect of the $\cos \phi$ Coriolis terms in the components of the momentum equation. If the spurious terms are retained, the resulting potential vorticity equation is

$$
\rho \frac{D_{a}}{D t}\left(\alpha \widetilde{\mathbf{Z}} \cdot \nabla_{a} \theta\right)+\left(\frac{4 \Omega \sin \phi}{a}\right) \mathbf{u} \cdot \nabla_{a} \theta+\frac{1}{a}\left(\nabla_{a} \times\left(w \mathbf{v}-\mathbf{k}\left(\mathbf{v}^{2}\right)\right)\right) \cdot \nabla_{a} \theta=R H S
$$

in which

$$
R H S=\nabla_{a} \cdot\left(\widetilde{\mathbf{Z}} \frac{D_{a} \theta}{D t}+\theta \nabla_{a} \times \mathbf{G}\right) .
$$

Of the two spurious terms on the left-hand side of (C.13), the one involving $\Omega$ sin $\phi$ necessarily vanishes in steady, isentropic flow (for which $D_{a} \theta / D t=\mathbf{u} \cdot \nabla_{a} \theta=0$ ), but the other remains even then.

White and Bromley (1995) deduced the PV conservation properties of the QHE model from the result in the NHD case by re-expressing $\nabla \times(\mathbf{k} D w / D t)$. The PV conservation law of the HPE model may be deduced from that of the NHS model by a parallel method. The shallow atmosphere equivalent of identity (4.6) of White and Bromley (1995) is

$$
\nabla_{a} \times\left(\mathbf{k} \frac{D_{a} w}{D t}\right)=\frac{D_{a} \Xi_{a}}{D t}+\Xi_{a} \nabla_{a} \cdot \mathbf{u}-\left(\boldsymbol{\Xi}_{a} \cdot \nabla_{a}\right) \mathbf{u}
$$

in which $\Xi_{a}$ is the part of the shallow atmosphere vorticity associated with vertical motion:

$$
\Xi_{a}=\left(\frac{1}{a} \frac{\partial w}{\partial \phi},-\frac{1}{a \cos \phi} \frac{\partial w}{\partial \lambda}, 0\right)
$$

Given (C.15), the PV conservation law of the HPEs follows easily from the NHS result. The effort here goes into substantiating (C.15); this involves analysis comparable in length to that of the direct proof of HPE PV conservation given in Appendix B. 


\section{TABLE 1}

SUMMARIZING THE APPROXIMATIONS MADE IN THE QUARTET OF MODELS DISCUSSED IN THIS PAPER, THE COORDINATES USED, METRIC FACTORS (SEE APPENDICES), DIFFERENTIAL OPERATORS,

MATERIAL DERIVATIVES AND QUANTITIES FEATURING IN THE CONSERVATION LAWS (SEE MAIN TEXT)

\begin{tabular}{|c|c|c|c|c|}
\hline & NHD model & QHEs & NHS model & HPEs \\
\hline $\begin{array}{l}\text { Spherical geopotential } \\
\text { approximation made? }\end{array}$ & Yes & "Yes & Yes & YYes \\
\hline $\begin{array}{l}\text { Dw/Dt omitted from vertical } \\
\text { component of momentum equation? }\end{array}$ & No & Yes & No & Yes \\
\hline $\begin{array}{l}\text { Shallow atmosphere } \\
\text { approximations made? }\end{array}$ & No & No & Yes & Yes \\
\hline Coordinates & \multicolumn{2}{|c|}{$(\lambda, \phi, r)$} & \multicolumn{2}{|c|}{$(\lambda, \phi, z)$} \\
\hline Metric factors & \multicolumn{2}{|c|}{$h_{\lambda}=r \cos \phi, \quad h_{\phi}=r, \quad h_{r}=1$} & \multicolumn{2}{|c|}{$h_{\lambda}=a \cos \phi, \quad h_{\phi}=a, \quad h_{z}=1$} \\
\hline grad & \multicolumn{2}{|c|}{$\nabla$ as given by $(3.2)$} & \multicolumn{2}{|c|}{$\nabla_{a}$ as given by $(3.21)$} \\
\hline $\operatorname{div} \mathbf{A}$ & \multicolumn{2}{|c|}{$\nabla \cdot \mathbf{A}$ as given by $(3.3)$} & \multicolumn{2}{|c|}{$\nabla_{a} \cdot \mathbf{A}$ as given by $(3.17)$} \\
\hline curlA & \multicolumn{2}{|c|}{$\nabla \times \mathbf{A}$ as given by $(3.4)$} & \multicolumn{2}{|c|}{$\nabla_{a} \times \mathbf{A}$ as given by $(3.22)$} \\
\hline Material derivative & \multicolumn{2}{|c|}{$D / D t=\partial / \partial t+\mathbf{u} \cdot \nabla$} & \multicolumn{2}{|c|}{$D_{a} / D t=\partial / \partial t+\mathbf{u} \cdot \nabla_{a}$} \\
\hline Axial absolute angular momentum & \multicolumn{2}{|c|}{$(u+\Omega r \cos \phi) r \cos \phi$} & \multicolumn{2}{|c|}{$(u+\Omega a \cos \phi) a \cos \phi$} \\
\hline Planetary vorticity & \multicolumn{2}{|c|}{$2 \Omega$} & \multicolumn{2}{|c|}{$f \mathbf{k}=2 \Omega \sin \phi \mathbf{k}$} \\
\hline Absolute vorticity & $\mathbf{Z}=2 \boldsymbol{\Omega}+\nabla \times \mathbf{u}$ & $\mathbf{Z}_{Q H E}=2 \boldsymbol{\Omega}+\nabla \times \mathbf{v}$ & $\mathbf{Z}_{N H S}=f \mathbf{k}+\nabla_{a} \times \mathbf{u}$ & $\mathbf{Z}_{H P E}=f \mathbf{k}+\nabla_{a} \times \mathbf{v}$ \\
\hline Potential vorticity & $\alpha \mathbf{Z} \cdot \nabla \theta$ & $\alpha \mathbf{Z}_{Q H E} \cdot \nabla \theta$ & $\alpha \mathbf{Z}_{N H S} \cdot \nabla_{a} \theta$ & $\alpha \mathbf{Z}_{H P E} \cdot \nabla_{a} \theta$ \\
\hline Total energy per unit mass & $\frac{1}{2} \mathbf{u}^{2}+\Phi_{A}(r)+c_{v} T$ & $\frac{1}{2} \mathbf{v}^{2}+\Phi_{A}(r)+c_{v} T$ & $\frac{1}{2} \mathbf{u}^{2}+g z+c_{v} T$ & $\frac{1}{2} \mathbf{v}^{2}+g z+c_{v} T$ \\
\hline
\end{tabular}




\section{Figure captions}

Figure 1. Polar section of a non-spherical geopotential surface, and directions of apparent vertical (arrows) normal to it. $\mathrm{O}$ is the centre of the Earth, which rotates with angular velocity $\Omega$ about the polar axis ON. OE lies in the equatorial plane. Apparent vertical is the direction of apparent gravity, and is locally delineated by a pendulum bob hanging at rest relative to the rotating Earth. At the poles and at the equator, the direction of apparent vertical coincides with the radii (ON and $\mathrm{OE}$ ) but at other (geocentric) latitudes $\phi$, centrifugal force leads to a deviation $\alpha$ of apparent vertical from the local radial direction. $\alpha$ reaches a maximum at about 45 degrees of latitude. For clarity, the diagram greatly exaggerates the geopotential eccentricity, typical tropospheric values of $\alpha$, and the distances of the points $\mathrm{E}$, $\mathrm{F}, \mathrm{G}, \mathrm{H}, \mathrm{N}$ from the geopotential.

Figure 2. The $\lambda, \phi, r$ spherical polar coordinate system. $\lambda$ is longitude, $\phi$ latitude of point $\mathrm{P}$ and $r$ its distance from the origin $\mathrm{O}$. The polar axis $\mathrm{ON}(\phi=\pi / 2)$ coincides with the rotation axis of the Earth. Arrows at $\mathrm{P}$ indicate the local unit vectors $\mathbf{i}, \mathbf{j}, \mathbf{k}$ associated with the corresponding $\lambda, \phi, r$ directions; velocity components $u, v, w$ are also shown.

Figure 3. Polar sections of two geopotentials treated as spheres centred at O. Apparent gravity is the gradient of a scalar field, so its line integral around any spatial circuit must vanish. It acts perpendicular to geopotentials, and in the spherical geopotential approximation is thus idealised as acting radially; the contributions to its line integral around circuit $\mathrm{ABCD}$ from the radial line segments $\mathrm{AB}$ and $\mathrm{CD}$ must therefore be equal and opposite. This will be so if apparent gravity is represented as varying only with radius (i.e. with distance from $\mathrm{O}$ ) but not, in general, if it is represented as varying also (or only) with latitude.

Figure 4. Showing the inter-relationships of the four consistent approximate models of the global atmosphere identified in this study (NHD, QHE, NHS and HPE models) and the relationship of the NHD model to the original (unapproximated) equations. G denotes the spherical geopotential approximation, $\mathbf{H}$ the omission of the term $D w / D t$ from the vertical component of the momentum equation, and $\mathbf{S}$ the shallow atmosphere combination of approximations (see text). 


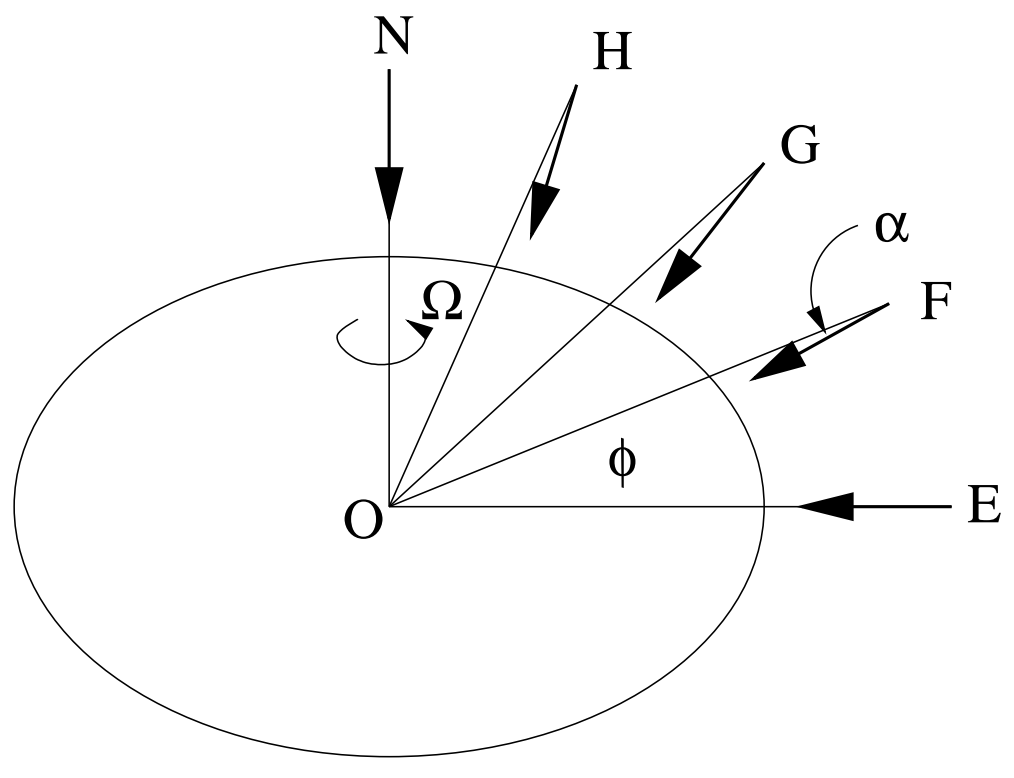

Figure 1: Polar section of a non-spherical geopotential surface, and directions of apparent vertical (arrows) normal to it. O is the centre of the Earth, which rotates with angular velocity $\Omega$ about the polar axis ON. OE lies in the equatorial plane. Apparent vertical is the direction of apparent gravity, and is locally delineated by a pendulum bob hanging at rest relative to the rotating Earth. At the poles and at the equator, the direction of apparent vertical coincides with the radii (ON and $\mathrm{OE}$ ) but at other (geocentric) latitudes $\phi$, centrifugal force leads to a deviation $\alpha$ of apparent vertical from the local radial direction. $\alpha$ reaches a maximum at about 45 degrees of latitude. For clarity, the diagram greatly exaggerates the geopotential eccentricity, typical tropospheric values of $\alpha$, and the distances of the points $\mathrm{E}, \mathrm{F}, \mathrm{G}, \mathrm{H}, \mathrm{N}$ from the geopotential. 


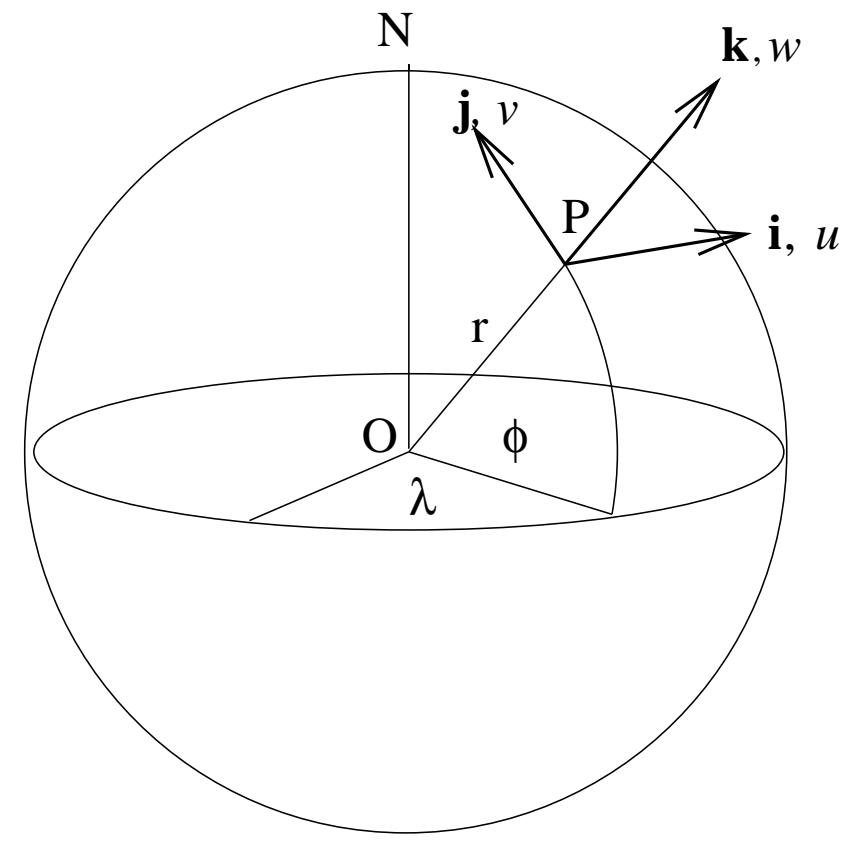

Figure 2: The $\lambda, \phi, r$ spherical polar coordinate system. $\lambda$ is longitude, $\phi$ latitude of point $\mathrm{P}$ and $r$ its distance from the origin $\mathrm{O}$. The polar axis $\mathrm{ON}(\phi=\pi / 2)$ coincides with the rotation axis of the Earth. Arrows at $\mathrm{P}$ indicate the local unit vectors $\mathbf{i}, \mathbf{j}, \mathbf{k}$ associated with the corresponding $\lambda, \phi, r$ directions; velocity components $u, v, w$ are also shown. 


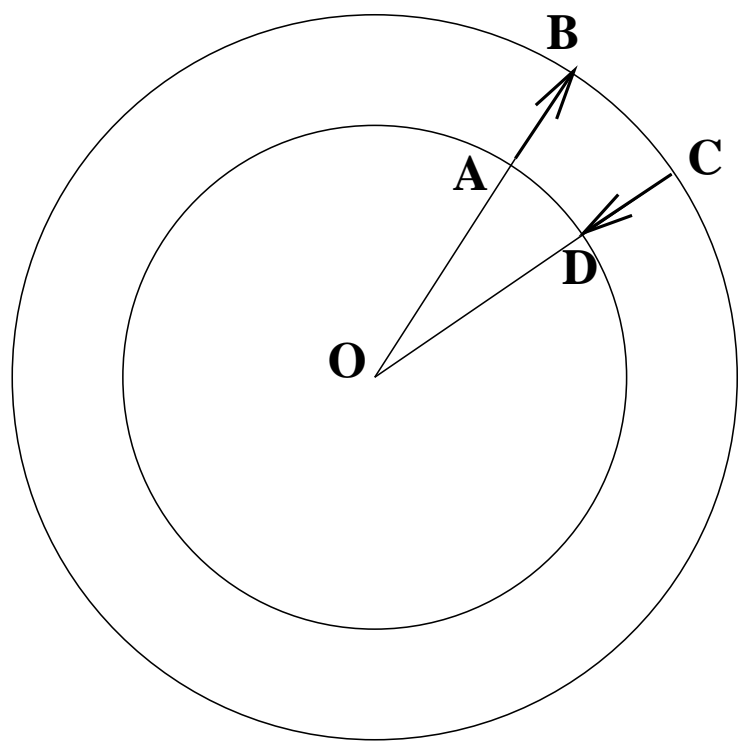

Figure 3: Polar sections of two geopotentials treated as spheres centred at O. Apparent gravity is the gradient of a scalar field, so its line integral around any spatial circuit must vanish. It acts perpendicular to geopotentials, and in the spherical geopotential approximation is thus idealised as acting radially; the contributions to its line integral around circuit ABCD from the radial line segments $\mathrm{AB}$ and $\mathrm{CD}$ must therefore be equal and opposite. This will be so if apparent gravity is represented as varying only with radius (i.e. with distance from O) but not, in general, if it is represented as varying also (or only) with latitude. 


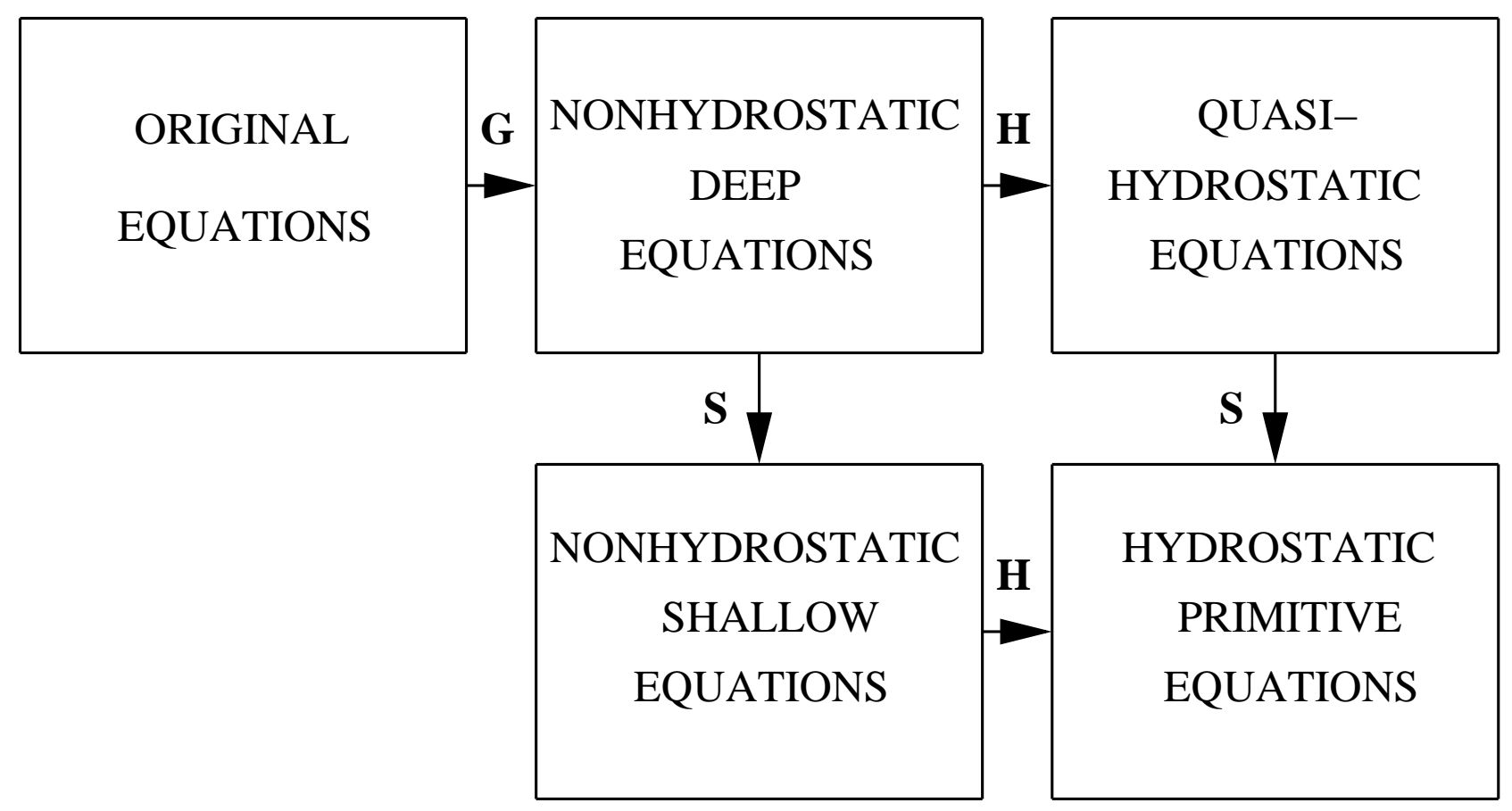

Figure 4: Showing the inter-relationships of the four consistent approximate models of the global atmosphere identified in this study (NHD, QHE, NHS and HPE models) and the relationship of the NHD model to the original (unapproximated) equations. $\mathbf{G}$ denotes the spherical geopotential approximation, $\mathbf{H}$ the omission of the term $D w / D t$ from the vertical component of the momentum equation, and $\mathbf{S}$ the shallow atmosphere combination of approximations (see text). 